Research Article

\title{
Perturbation Methods for the Eigencharacteristics of Symmetric and Asymmetric Systems
}

\author{
Philip D. Cha (iD) and Austin Shin \\ Department of Engineering, Harvey Mudd College, 301 Platt Blvd., Claremont, CA 91711, USA \\ Correspondence should be addressed to Philip D. Cha; philip_cha@hmc.edu
}

Received 9 May 2018; Accepted 28 August 2018; Published 17 October 2018

Academic Editor: Marcello Vanali

Copyright (c) 2018 Philip D. Cha and Austin Shin. This is an open access article distributed under the Creative Commons Attribution License, which permits unrestricted use, distribution, and reproduction in any medium, provided the original work is properly cited.

\begin{abstract}
Dynamic analysis for a vibratory system typically begins with an evaluation of its eigencharacteristics. However, when design changes are introduced, the eigensolutions of the system change and thus must be recomputed. In this paper, three different methods based on the eigenvalue perturbation theory are introduced to analyze the effects of modifications without performing a potentially time-consuming and costly reanalysis. They will be referred to as the straightforward perturbation method, the incremental perturbation method, and the triple product method. In the straightforward perturbation method, the eigenvalue perturbation theory is used to formulate a first-order and a second-order approximation of the eigensolutions of symmetric and asymmetric systems. In the incremental perturbation method, the straightforward approach is extended to analyze systems with large perturbations using an iterative scheme. Finally, in the triple product method, the accuracy of the approximate eigenvalues is significantly improved by exploiting the orthogonality conditions of the perturbed eigenvectors. All three methods require only the eigensolutions of the nominal or unperturbed system, and in application, they involve simple matrix multiplications. Numerical experiments show that the proposed methods achieve accurate results for systems with and without damping and for systems with symmetric and asymmetric system matrices.
\end{abstract}

\section{Introduction}

In order to analyze the vibration of any structural system, one usually first calculates the system's eigensolutions. After the initial analysis has been performed, if any design modifications are then subsequently introduced, one would need to recalculate the perturbed system's eigensolutions. However, when the degrees-of-freedom for the system is large, solving the eigenvalue problem multiple times for each set of perturbations can be computationally expensive and undesirable. In these cases, eigenvalue perturbation theory can be applied to obtain the approximate eigencharacteristics of the system without resolving an entirely new eigenvalue problem. The expressions for the approximate eigensolutions of the perturbed system consist solely of matrix multiplications, which can be efficiently performed by any computer.
Over the years, there has been a large amount of interest in applying the perturbation theory and sensitivity analysis to study the effects of design changes on the eigensolutions of structures. Because of the large number of studies that have been published in these areas, only selected references will be addressed in this paper. Fox and Kapoor [1] derived expressions for the first derivatives of eigenvalues and eigenvectors with respect to a system's parameters. The expressions were applicable only to self-adjoint systems, and the numerical examples used only contained real eigenvalues and were restricted to small perturbations in the system. Romstad et al. [2] formulated a power-series perturbation approach and applied the method to structural systems in order to find perturbed distinct or repeated eigenvalues and eigenvalue sensitivities. However, the systems they analyzed were symmetric and undamped. Plaut and Huseyin [3] derived expressions of the derivatives of eigenvalues and eigenvectors of non-self-adjoint systems. Rudisill [4] further 
explored sensitivity analysis by deriving simpler expressions of a system's eigenvalues and eigenvectors using only one left-hand and right-hand eigenvector. These expressions could also be extended to any order of derivative of the eigenvalue and eigenvector, though it was not explicitly done so in the paper. Meirovitch $[5,6]$ obtained the complete response of gyroscopic systems using the perturbation method, but his method required an extra step of reducing the generalized eigenvalue problem to its standard form in order to take advantage of the fact that the gyroscopic matrix is skew symmetric. Rudisill and Chu [7] built upon the work of Rudisill and introduced two numerical methods of computing the derivatives of a system's eigenvalues and eigenvectors. The first method consisted of an iterative method that could be used to compute the first partial derivatives of the eigenvalues and eigenvectors of a selfadjoint system and only the first partial derivative of the largest eigenvalue and its associated eigenvector of a nonself-adjoint system. The second method was an algebraic method that could be used to compute all orders of the eigensolutions' derivatives without using the left-hand eigenvectors. However, unlike in Rudisill's work, this method required the solution of $(n+1)$ equations as opposed to simply $n$ equations. Nelson [8] presented a simplified procedure of calculating eigenvector derivatives of an eigensystem of any order using only the left and right eigenvectors and their associated eigenvalues. However, these expressions were derived based on only the standard eigenvalue problem, and the original eigensystem's matrix of rank $(n-1)$ must be converted to rank $n$. Chen and Wada [9] formulated a first-order perturbation solution using a power-series expansion to conduct structural dynamic analysis. However, their analysis was restricted to systems with symmetric matrices. Meirovitch and Ryland [10] examined gyroscopic systems with small internal damping and utilized a second-order approach along with the Cholesky decomposition and linear transformations to reduce the generalized eigenvalue problem to a standard one. Because the damping considered was small, they used a perturbation approach to obtain the response of the system by considering the undamped gyroscopic system as the unperturbed system and treated the damping as perturbation. Belle [11] presented expressions of higher order sensitivities, allowing for more accurate approximations of a perturbed system's eigensolutions than when using only the first partial derivatives. However, the paper did not quantify the improvement in accuracy with these higher-order sensitivities. Meirovitch and Ryland [12] extended their aforementioned perturbation approach to include external damping and used Rayleigh's quotient to enhance the accuracy of the approximate eigenvalues used in the modal analysis. Baldwin and Hutton [13] surveyed various structural dynamics modification techniques, and in their analysis of perturbation techniques, they described a first-order approach restricted to systems without any damping. Chung and Lee [14] developed a second-order perturbation solution and analyzed heavily but weakly proportionally damped systems. Bickford [15] proposed a scheme that improved the accuracy of the approximate eigenvalues obtained using the usual first-order perturbation approach. It led to results whose accuracy approached those using the second-order perturbation approach. The method, however, was strictly valid for symmetric eigenvalue problems. Cronin [16] used the perturbation approach to analyze nonclassically damped dynamic systems. The proposed method involved a partial diagonalization of the homogeneous equations of motion. A perturbation quantity based on the off-diagonal terms of the partially diagonalized damping matrix was then defined. The eigenvalues and eigenvectors for the damped system were described in terms of power-series in the perturbation quantity. Wang [17] introduced two approximate methods for calculating a eigenvector's derivatives when only a truncated set of the system's mode shapes is available. The first was the explicit method, which approximated the contribution of higher modes. The second method was the implicit method, which assumed that the eigenvector derivatives were spanned by the truncated mode shapes and a residual static mode. Eldred et al. [18] developed an improved mass normalization method for expressing eigenvalue derivatives of structural systems. This improved method performed accurately only for slightly less restrictive parameter changes (up to 10\%). In addition, Eldred et al. [19] explored the perturbation approach and developed a solution of the full nonlinear eigensolution perturbation equations that took into account all orders of the perturbation expansion, whereby the frequency and mode shape perturbation equations were coupled. The method presented in this paper will show that these coupled equations are not necessary in order to achieve accurate results with large perturbations. Kwak [20] proposed a power-series perturbation approach to express the perturbed eigensolutions of lightly damped systems. However, the analysis depended on the original system being undamped. Chen et al. [21] extended the perturbation approach to not only deal with distinct or repeated eigenvalues but also close eigenvalues or eigenvalue clusters. Their analysis, however, was constrained to deal with relatively small perturbations, and the system they analyzed contained only symmetric system matrices and no damping. Wang and Kirkhope [22] used a perturbation approach to analyze damped rotor systems. The solution scheme they developed, however, was restricted to gyroscopic systems with no cross-coupling other than gyroscopic effects. Tang and Wang [23] applied a power-series perturbation approach to analyze systems with distinct, repeated, and nearly equal frequencies. Their technique utilized complex operations such as subspace condensation and orthogonal decompositions, and they only considered undamped systems with small perturbations. Liu and Chan [24] developed an efficient matrix perturbation technique to approximate the changes in the eigensolutions of the system due to structural modifications. The solution scheme required one to perform a subspace condensation and an orthogonal decomposition in order to obtain the lowerorder perturbed eigensolutions. The matrix singular value decomposition was then subsequently used to compute the higher-order perturbations of the eigensolutions. Trisovic [25] applied sensitivity analysis to two structural systems, a shaft of an electromotor and a cantilever beam, utilizing 
only the first partial derivatives of the eigenvalues and eigenvectors to alter the systems' natural frequencies. Cha and Solberg [26] applied the eigenvalue perturbation theory to analyze various problems in structural dynamics. The method they used, however, was restricted to a first-order analysis and only valid for symmetric systems. ElBeheiry [27] analyzed weakly, nonproportionally damped systems, where this damping stemmed from gyroscopic moments or external damping factors. He utilized a second-order perturbation approach and represented the original system as a conservative gyroscopic system. However, the perturbations in the system were restricted to be one order of magnitude smaller than the values in the original system matrices.

In this paper, simple methods are introduced that can be used to obtain the approximate eigenvalues and eigenvectors of various systems, including those with asymmetric mass, stiffness, or damping matrices and those with varying magnitudes of perturbations. A simple first-order perturbation analysis is sufficient if the modifications introduced into the system are small. By including perturbations up to the second-order, the accuracy achieved with the straightforward method becomes better than that obtained using a first-order perturbation approach. When the design changes are sufficiently large, the straightforward perturbation method, even with a second-order analysis, fails to approximate the structural vibration eigencharacteristics. In this case, the incremental perturbation method can be employed, which returns accurate eigensolutions because the system is minimally perturbed from one iteration to the next. Finally, a simple triple product method is proposed that further enhances the accuracy of the approximate eigenvalues of the modified systems. Numerical examples are presented to demonstrate the accuracy of the proposed perturbation methods for undamped and damped systems, and for structures with symmetric and asymmetric system matrices.

\section{Theory}

The use of eigenvalue perturbation theory in the design of mechanical systems has been well explored. However, most papers have only applied this method to systems with small damping parameters or to systems with symmetric mass and stiffness matrices. In the following, the first-order and second-order approximations of the perturbed eigencharacteristics are derived based on the perturbation theory. This formulation can be applied to systems with asymmetric matrices, such as gyroscopic systems, and to systems with large damping. An iterative scheme based on perturbing the system matrices incrementally is also proposed, which allows one to obtain accurate approximations of the eigensolutions of the perturbed system when large modifications are introduced. Finally, knowing the perturbed eigenvectors, a third approach is developed that yields approximate eigenvalues that are more accurate than the previous methods.
2.1. Straightforward Perturbation Method. Consider a system whose modes of vibration are governed by the solutions of the following generalized eigenvalue problem:

$$
\left[B_{0}\right] \mathbf{x}_{0}=\lambda_{0}\left[A_{0}\right] \mathbf{x}_{0},
$$

where $\left[B_{0}\right]$ and $\left[A_{0}\right]$ denote the nominal system matrices, both of size $p \times p, \lambda_{0}$ represents a nominal eigenvalue, and $\mathbf{x}_{0}$ is the corresponding nominal right eigenvector. Assume all the unperturbed eigenvalues of the system are distinct, and $\left[B_{0}\right]$ and $\left[A_{0}\right]$ are asymmetric. The initial system's left eigenvectors, denoted by $\mathbf{y}_{0}$, satisfy

$$
\left[B_{0}\right]^{T} \mathbf{y}_{0}=\lambda_{0}\left[A_{0}\right]^{T} \mathbf{y}_{0}
$$

Assume the $j$ th left eigenvector and the $i$ th right eigenvector have been properly normalized such that they satisfy the following orthogonality conditions:

$$
\begin{aligned}
& \mathbf{y}_{0 j}^{T}\left[A_{0}\right] \mathbf{x}_{0 i}=\delta_{i}^{j}, \\
& \mathbf{y}_{0 j}^{T}\left[B_{0}\right] \mathbf{x}_{0 i}=\lambda_{0 i} \delta_{i}^{j},
\end{aligned}
$$

where $\delta_{i}^{j}$ denotes the Kronecker delta and $i, j=1, \ldots, p$.

After an initial analysis has been performed, design changes are introduced so that the system matrices become

$$
\begin{aligned}
& {[A]=\left[A_{0}\right]+[\delta A],} \\
& {[B]=\left[B_{0}\right]+[\delta B],}
\end{aligned}
$$

where $\left[A_{0}\right]$ and $\left[B_{0}\right]$ denote the unperturbed matrices, $[\delta A]$ and $[\delta B]$ the perturbation matrices, and $[A]$ and $[B]$ the perturbed matrices. Moreover, elements of $[\delta A]$ and $[\delta B]$ are assumed to be an order of magnitude smaller than those of $\left[A_{0}\right]$ and $\left[B_{0}\right]$. Because the system parameters have been perturbed, the eigensolutions of the modified system will undergo changes as follows:

$$
\begin{aligned}
& \lambda_{j}=\lambda_{0 j}+\delta \lambda_{j}+\delta^{2} \lambda_{j}, \\
& \mathbf{x}_{j}=\mathbf{x}_{0 j}+\delta \mathbf{x}_{j}+\delta^{2} \mathbf{x}_{j}, \\
& \mathbf{y}_{j}=\mathbf{y}_{0 j}+\delta \mathbf{y}_{j}+\delta^{2} \mathbf{y}_{j},
\end{aligned}
$$

where $\delta \lambda_{j}, \delta \mathbf{x}_{j}$, and $\delta \mathbf{y}_{j}$ denote the first-order eigensolution perturbations, and $\delta^{2} \lambda_{j}, \delta^{2} \mathbf{x}_{j}$, and $\delta^{2} \mathbf{y}_{j}$ represent the secondorder eigensolution perturbations. Resolving the eigenvalue problem associated with the perturbed system can be timeconsuming and computationally taxing. Instead, the perturbation theory is used to find the approximate eigencharacteristics of the modified system. For completeness, the eigenvalue perturbation theory will be presented in detail.

Consider the $j$ th right and left eigenvectors of the perturbed system that satisfy

$$
\begin{aligned}
{[B] \mathbf{x}_{j} } & =\lambda_{j}[A] \mathbf{x}_{j}, \\
\mathbf{y}_{j}^{T}[B] & =\lambda_{j} \mathbf{y}_{j}^{T}[A] .
\end{aligned}
$$

Substituting Equations (4) and (5) in Equation (6), expanding, and keeping only the first-order and secondorder terms, the following equations are obtained: 


$$
\begin{aligned}
{\left[B_{0}\right] \mathbf{x}_{0 j}+\left[B_{0}\right] \delta \mathbf{x}_{j}+\left[B_{0}\right] \delta^{2} \mathbf{x}_{j}+[\delta B] \mathbf{x}_{0 j}+[\delta B] \delta \mathbf{x}_{j} } \\
=\lambda_{0 j}\left[A_{0}\right] \mathbf{x}_{0 j}+\lambda_{0 j}\left[A_{0}\right] \delta \mathbf{x}_{j}+\lambda_{0 j}\left[A_{0}\right] \delta^{2} \mathbf{x}_{j} \\
+\lambda_{0 j}[\delta A] \mathbf{x}_{0 j}+\lambda_{0 j}[\delta A] \delta \mathbf{x}_{j}+\delta \lambda_{j}\left[A_{0}\right] \mathbf{x}_{0 j} \\
+\delta \lambda_{j}\left[A_{0}\right] \delta \mathbf{x}_{j}+\delta \lambda_{j}[\delta A] \mathbf{x}_{0 j}+\delta^{2} \lambda_{j}\left[A_{0}\right] \mathbf{x}_{0 j}, \\
\mathbf{y}_{0 j}^{T}\left[B_{0}\right]+\delta \mathbf{y}_{j}^{T}\left[B_{0}\right]+\delta^{2} \mathbf{y}_{j}^{T}\left[B_{0}\right]+\mathbf{y}_{0 j}^{T}[\delta B]+\delta \mathbf{y}_{j}^{T}[\delta B] \\
=\lambda_{0 j} \mathbf{y}_{0 j}^{T}\left[A_{0}\right]+\lambda_{0 j} \delta \mathbf{y}_{j}^{T}\left[A_{0}\right]+\lambda_{0 j} \delta^{2} \mathbf{y}_{j}^{T}\left[A_{0}\right] \\
+\lambda_{0 j} \mathbf{y}_{0 j}^{T}[\delta A]+\lambda_{0 j} \delta \mathbf{y}_{j}^{T}[\delta A]+\delta \lambda_{j} \mathbf{y}_{0 j}^{T}\left[A_{0}\right] \\
+\delta \lambda_{j} \delta \mathbf{y}_{j}^{T}\left[A_{0}\right]+\delta \lambda_{j} \mathbf{y}_{0 j}^{T}[\delta A]+\delta^{2} \lambda_{j} \mathbf{y}_{0 j}^{T}\left[A_{0}\right] .
\end{aligned}
$$
gives

Equating the first-order terms in Equations (7) and (8)

$$
\left[B_{0}\right] \delta \mathbf{x}_{j}+[\delta B] \mathbf{x}_{0 j}=\lambda_{0 j}\left[A_{0}\right] \delta \mathbf{x}_{j}+\lambda_{0 j}[\delta A] \mathbf{x}_{0 j}+\delta \lambda_{j}\left[A_{0}\right] \mathbf{x}_{0 j},
$$

$\mathbf{y}_{0 j}^{T}[\delta B]+\delta \mathbf{y}_{j}^{T}\left[B_{0}\right]=\lambda_{0 j} \mathbf{y}_{0 j}^{T}[\delta A]+\lambda_{0 j} \delta \mathbf{y}_{j}^{T}\left[A_{0}\right]+\delta \lambda_{j} \mathbf{y}_{0 j}^{T}\left[A_{0}\right]$

Similarly, equating the second-order terms in Equations (7) and (8) yields

$$
\begin{aligned}
{\left[B_{0}\right] \delta^{2} \mathbf{x}_{j}+[\delta B] \delta \mathbf{x}_{j}=} & \lambda_{0 j}\left[A_{0}\right] \delta^{2} \mathbf{x}_{j}+\lambda_{0 j}[\delta A] \delta \mathbf{x}_{j} \\
& +\delta \lambda_{j}\left[A_{0}\right] \delta \mathbf{x}_{j}+\delta \lambda_{j}[\delta A] \mathbf{x}_{0 j} \\
& +\delta^{2} \lambda_{j}\left[A_{0}\right] \mathbf{x}_{0 j}, \\
\delta \mathbf{y}_{j}^{T}[\delta B]+\delta^{2} \mathbf{y}_{j}^{T}\left[B_{0}\right]= & \lambda_{0 j} \delta \mathbf{y}_{j}^{T}[\delta A]+\lambda_{0 j} \delta^{2} \mathbf{y}_{j}^{T}\left[A_{0}\right] \\
& +\delta \lambda_{j} \mathbf{y}_{0 j}^{T}[\delta A]+\delta \lambda_{j} \delta \mathbf{y}_{j}^{T}\left[A_{0}\right] \\
& +\delta^{2} \lambda_{j} \mathbf{y}_{0 j}^{T}\left[A_{0}\right] .
\end{aligned}
$$

Because the unperturbed eigenvalues are distinct, the unperturbed eigenvectors are linearly independent and form a basis. Thus, $\delta \mathbf{x}_{j}$ and $\delta^{2} \mathbf{x}_{j}$ can be expressed as a linear combination of $\mathbf{x}_{0 r}$ as follows:

$$
\begin{gathered}
\delta \mathbf{x}_{j}=\sum_{r=1}^{p} \varepsilon_{r j} \mathbf{x}_{0 r}, \\
\delta^{2} \mathbf{x}_{j}=\sum_{r=1}^{p} \eta_{r j} \mathbf{x}_{0 r},
\end{gathered}
$$

where $\varepsilon_{r j}$ and $\eta_{r j}$ are small coefficients that are to be determined.

Consider Equation (9), which consists of the firstorder terms. Substituting Equation (13) in Equation (9) gives

$$
\begin{aligned}
{\left[B_{0}\right] \sum_{r=1}^{p} \varepsilon_{r j} \mathbf{x}_{0 r}+[\delta B] \mathbf{x}_{0 j}=} & \lambda_{0 j}\left[A_{0}\right] \sum_{r=1}^{p} \varepsilon_{r j} \mathbf{x}_{0 r} \\
& +\lambda_{0 j}[\delta A] \mathbf{x}_{0 j}+\delta \lambda_{j}\left[A_{0}\right] \mathbf{x}_{0 j} .
\end{aligned}
$$

Premultiplying Equation (15) by $\mathbf{y}_{0 j}^{T}$ and noting the orthogonality conditions of Equation (3), one finds an expression for $\delta \lambda_{j}$, the first-order eigenvalue perturbation, as follows:

$$
\delta \lambda_{j}=\mathbf{y}_{0 j}^{T}\left([\delta B]-\lambda_{0 j}[\delta A]\right) \mathbf{x}_{0 j} .
$$

Premultiplying Equation (9) by $\mathbf{y}_{0 i}^{T}$, where $i \neq j$, one can readily solve for $\varepsilon_{i j}$ :

$$
\varepsilon_{i j}=\frac{\mathbf{y}_{0 i}^{T}\left([\delta B]-\lambda_{0 j}[\delta A]\right) \mathbf{x}_{0 j}}{\lambda_{0 j}-\lambda_{0 i}} .
$$

Consider now Equation (11), which consists of the second-order terms. Substituting Equation (14) in Equation (11) gives

$$
\begin{aligned}
{\left[B_{0}\right] \sum_{r=1}^{p} \eta_{r j} \mathbf{x}_{0 r}+[\delta B] \delta \mathbf{x}_{j}=} & \lambda_{0 j}\left[A_{0}\right] \sum_{r=1}^{p} \eta_{r j} \mathbf{x}_{0 r} \\
& +\lambda_{0 j}[\delta A] \delta \mathbf{x}_{j}+\delta \lambda_{j}\left[A_{0}\right] \delta \mathbf{x}_{j} \\
& +\delta \lambda_{j}[\delta A] \mathbf{x}_{0 j}+\delta^{2} \lambda_{j}\left[A_{0}\right] \mathbf{x}_{0 j} .
\end{aligned}
$$

Premultiplying Equation (18) by $\mathbf{y}_{0 j}^{T}$ and exploiting the orthogonality conditions, one obtains the following expression for $\delta^{2} \lambda_{j}$, the second-order eigenvalue perturbation: $\delta^{2} \lambda_{j}=\mathbf{y}_{0 j}^{T}\left([\delta B]-\lambda_{0 j}[\delta A]\right) \delta \mathbf{x}_{j}-\delta \lambda_{j} \mathbf{y}_{0 j}^{T}\left(\left[A_{0}\right] \delta \mathbf{x}_{j}+[\delta A] \mathbf{x}_{0 j}\right)$.

Premultiplying Equation (18) by $\mathbf{y}_{0 i}^{T}$, for $i \neq j$, one obtains $\eta_{i j}=\frac{\mathbf{y}_{0 i}^{T}\left([\delta B]-\lambda_{0 j}[\delta A]\right) \delta \mathbf{x}_{j}-\delta \lambda_{j} \mathbf{y}_{0 i}^{T}\left(\left[A_{0}\right] \delta \mathbf{x}_{j}+[\delta A] \mathbf{x}_{0 j}\right)}{\lambda_{0 j}-\lambda_{0 i}}$.

To determine $\varepsilon_{j j}$ and $\eta_{j j}$, consider

$$
\mathbf{y}_{j}^{T}[A] \mathbf{x}_{j}=1 \text {. }
$$

Substituting Equations (4) and (5) in Equation (21), expanding the resulting expression, and keeping only terms up to the second-order, one has

$$
\begin{aligned}
\mathbf{y}_{0 j}^{T} & {\left[A_{0}\right] \delta \mathbf{x}_{j}+\mathbf{y}_{0 j}^{T}\left[A_{0}\right] \delta^{2} \mathbf{x}_{j}+\mathbf{y}_{0 j}^{T}[\delta A] \mathbf{x}_{0 j} } \\
& +\mathbf{y}_{0 j}^{T}[\delta A] \delta \mathbf{x}_{j}+\delta \mathbf{y}_{j}^{T}\left[A_{0}\right] \mathbf{x}_{0 j}+\delta \mathbf{y}_{j}^{T}\left[A_{0}\right] \delta \mathbf{x}_{j} \\
& +\delta \mathbf{y}_{j}^{T}[\delta A] \mathbf{x}_{0 j}+\delta^{2} \mathbf{y}_{j}^{T}\left[A_{0}\right] \mathbf{x}_{0 j}=0 .
\end{aligned}
$$

Equating the first-order and second-order terms in Equation (22) gives

$$
\begin{aligned}
& \mathbf{y}_{0 j}^{T}\left[A_{0}\right] \delta \mathbf{x}_{j}+\mathbf{y}_{0 j}^{T}[\delta A] \mathbf{x}_{0 j}+\delta \mathbf{y}_{j}^{T}\left[A_{0}\right] \mathbf{x}_{0 j}=0, \\
& \mathbf{y}_{0 j}^{T}\left[A_{0}\right] \delta^{2} \mathbf{x}_{j}+\mathbf{y}_{0 j}^{T}[\delta A] \delta \mathbf{x}_{j} \\
& \quad+\delta \mathbf{y}_{j}^{T}\left[A_{0}\right] \delta \mathbf{x}_{j}+\delta \mathbf{y}_{j}^{T}[\delta A] \mathbf{x}_{0 j}+\delta^{2} \mathbf{y}_{j}^{T}\left[A_{0}\right] \mathbf{x}_{0 j}=0 .
\end{aligned}
$$

Expressing $\delta \mathbf{y}_{j}$ and $\delta^{2} \mathbf{y}_{j}$ as a linear combination of $\mathbf{y}_{0 r}$, one obtains 


$$
\begin{aligned}
\delta \mathbf{y}_{j} & =\sum_{r=1}^{p} \widehat{\varepsilon}_{r j} \mathbf{y}_{0 r}, \\
\delta^{2} \mathbf{y}_{j} & =\sum_{r=1}^{p} \widehat{\eta}_{r j} \mathbf{y}_{0 r} .
\end{aligned}
$$

Substituting Equations (13) and (25) in Equation (23) gives

$$
\mathbf{y}_{0 j}^{T}\left[A_{0}\right] \sum_{r=1}^{p} \varepsilon_{r j} \mathbf{x}_{0 r}+\mathbf{y}_{0 j}^{T}[\delta A] \mathbf{x}_{0 j}+\left(\sum_{r=1}^{p} \widehat{\varepsilon}_{r j} \mathbf{y}_{0 r}^{T}\right)\left[A_{0}\right] \mathbf{x}_{0 j}=0,
$$

and because of the orthogonality conditions, Equation (27) reduces to

$$
\varepsilon_{j j}+\mathbf{y}_{0 j}^{T}[\delta A] \mathbf{x}_{0 j}+\widehat{\varepsilon}_{j j}=0
$$

Assuming $\varepsilon_{j j}=\widehat{\varepsilon}_{j j}$, then

$$
\varepsilon_{j j}=\widehat{\varepsilon}_{j j}=-\frac{1}{2} \mathbf{y}_{0 j}^{T}[\delta A] \mathbf{x}_{0 j}
$$

Substituting Equations (14) and (26) in Equation (24) leads to

$$
\begin{array}{r}
\mathbf{y}_{0 j}^{T}\left[A_{0}\right] \sum_{r=1}^{p} \eta_{r j} \mathbf{x}_{0 r}+\mathbf{y}_{0 j}^{T}[\delta A] \delta \mathbf{x}_{j}+\delta \mathbf{y}_{j}^{T}\left[A_{0}\right] \delta \mathbf{x}_{j} \\
+\delta \mathbf{y}_{j}^{T}[\delta A] \mathbf{x}_{0 j}+\left(\sum_{r=1}^{p} \widehat{\eta}_{r j} \mathbf{y}_{0 r}^{T}\right)\left[A_{0}\right] \mathbf{x}_{0 j}=0
\end{array}
$$

which simplifies to

$$
\eta_{j j}+\mathbf{y}_{0 j}^{T}[\delta A] \delta \mathbf{x}_{j}+\delta \mathbf{y}_{j}^{T}\left[A_{0}\right] \delta \mathbf{x}_{j}+\delta \mathbf{y}_{j}^{T}[\delta A] \mathbf{x}_{0 j}+\widehat{\eta}_{j j}=0,
$$
then

due to the orthogonality conditions. Assuming $\eta_{j j}=\widehat{\eta}_{j j}$,

$$
\eta_{j j}=\widehat{\eta}_{j j}=-\frac{1}{2}\left(\mathbf{y}_{0 j}^{T}[\delta A] \delta \mathbf{x}_{j}+\delta \mathbf{y}_{j}^{T}\left[A_{0}\right] \delta \mathbf{x}_{j}+\delta \mathbf{y}_{j}^{T}[\delta A] \mathbf{x}_{0 j}\right) .
$$

In summary, the perturbed eigenvalues are given by

$$
\begin{aligned}
\lambda_{j}= & \lambda_{0 j}+\delta \lambda_{j}+\delta^{2} \lambda_{j}, \\
\delta \lambda_{j}= & \mathbf{y}_{0 j}^{T}\left([\delta B]-\lambda_{0 j}[\delta A]\right) \mathbf{x}_{0 j}, \\
\delta^{2} \lambda_{j}= & \mathbf{y}_{0 j}^{T}\left([\delta B]-\lambda_{0 j}[\delta A]\right) \delta \mathbf{x}_{j} \\
& -\delta \lambda_{j} \mathbf{y}_{0 j}^{T}\left(\left[A_{0}\right] \delta \mathbf{x}_{j}+[\delta A] \mathbf{x}_{0 j}\right) .
\end{aligned}
$$

The perturbed right eigenvectors are

$$
\begin{aligned}
\mathbf{x}_{j} & =\mathbf{x}_{0 j}+\delta \mathbf{x}_{j}+\delta^{2} \mathbf{x}_{j}, \\
\delta \mathbf{x}_{j} & =\sum_{r=1}^{p} \varepsilon_{r j} \mathbf{x}_{0 r}, \\
\delta^{2} \mathbf{x}_{j} & =\sum_{r=1}^{p} \eta_{r j} \mathbf{x}_{0 r}, \\
\varepsilon_{i j} & =\frac{\mathbf{y}_{0 i}^{T}\left([\delta B]-\lambda_{0 j}[\delta A]\right) \mathbf{x}_{0 j}}{\lambda_{0 j}-\lambda_{0 i}} \text { for } i \neq j, \\
\varepsilon_{j j} & =-\frac{1}{2} \mathbf{y}_{0 j}^{T}[\delta A] \mathbf{x}_{0 j}, \\
\eta_{i j} & =\frac{\mathbf{y}_{0 i}^{T}\left([\delta B]-\lambda_{0 j}[\delta A]\right) \delta \mathbf{x}_{j}-\delta \lambda_{j} \mathbf{y}_{0 i}^{T}\left(\left[A_{0}\right] \delta \mathbf{x}_{j}+[\delta A] \mathbf{x}_{0 j}\right)}{\lambda_{0 j}-\lambda_{0 i}}
\end{aligned}
$$

for $i \neq j$,

$$
\eta_{j j}=-\frac{1}{2}\left(\mathbf{y}_{0 j}^{T}[\delta A] \delta \mathbf{x}_{j}+\delta \mathbf{y}_{j}^{T}\left[A_{0}\right] \delta \mathbf{x}_{j}+\delta \mathbf{y}_{j}^{T}[\delta A] \mathbf{x}_{0 j}\right),
$$

and the perturbed left eigenvectors are

$$
\begin{aligned}
\mathbf{y}_{j} & =\mathbf{y}_{0 j}+\delta \mathbf{y}_{j}+\delta^{2} \mathbf{y}_{j}, \\
\delta \mathbf{y}_{j} & =\sum_{r=1}^{p} \widehat{\varepsilon}_{r j} \mathbf{y}_{0 r}, \\
\delta^{2} \mathbf{y}_{j} & =\sum_{r=1}^{p} \widehat{\eta}_{r j} \mathbf{y}_{0 r}, \\
\widehat{\varepsilon}_{i j} & =\frac{\mathbf{y}_{0 j}^{T}\left([\delta B]-\lambda_{0 j}[\delta A]\right) \mathbf{x}_{0 i}}{\lambda_{0 j}-\lambda_{0 i}} \text { for } i \neq j, \\
\widehat{\varepsilon}_{j j} & =\varepsilon_{j j}, \\
\widehat{\eta}_{i j} & =\frac{\mathbf{y}_{0 j}^{T}\left([\delta B]-\lambda_{0 j}[\delta A]\right) \delta \mathbf{x}_{i}-\delta \lambda_{j} \mathbf{y}_{0 j}^{T}\left(\left[A_{0}\right] \delta \mathbf{x}_{i}+[\delta A] \mathbf{x}_{0 i}\right)}{\lambda_{0 j}-\lambda_{0 i}}
\end{aligned}
$$

for $i \neq j$,

$$
\widehat{\eta}_{j j}=\eta_{j j}
$$

Equations (33)-(35) can be used to obtain the approximate first-order or the second-order eigencharacteristics of an asymmetric system whose system parameters have undergone design changes or modifications. For a first-order analysis, the perturbed eigensolutions are given by 


$$
\begin{aligned}
& \lambda_{j}=\lambda_{0 j}+\delta \lambda_{j}, \\
& \mathbf{x}_{j}=\mathbf{x}_{0 j}+\delta \mathbf{x}_{j}, \\
& \mathbf{y}_{j}=\mathbf{y}_{0 j}+\delta \mathbf{y}_{j},
\end{aligned}
$$

and for a second-order analysis, the perturbed eigensolutions are given by

$$
\begin{aligned}
& \lambda_{j}=\lambda_{0 j}+\delta \lambda_{j}+\delta^{2} \lambda_{j}, \\
& \mathbf{x}_{j}=\mathbf{x}_{0 j}+\delta \mathbf{x}_{j}+\delta^{2} \mathbf{x}_{j}, \\
& \mathbf{y}_{j}=\mathbf{y}_{0 j}+\delta \mathbf{y}_{j}+\delta^{2} \mathbf{y}_{j} .
\end{aligned}
$$

While these equations appear to be complicated at first glance, they are easy to code and efficient to run because they only involve simple matrix multiplications. Finally, for a symmetric system where $\left[A_{0}\right]=\left[A_{0}\right]^{T}$ and $\left[B_{0}\right]=\left[B_{0}\right]^{T}$, the left and right eigenvectors are identical, i.e., $\mathbf{y}_{j}=\mathbf{x}_{j}$. Thus, in applying the perturbation equations, only the perturbed right eigenvectors need to be solved.

2.2. Incremental Perturbation Method. While the perturbation method is applicable only when elements of $[\delta A]$ and $[\delta B]$ are assumed to be an order of magnitude smaller than those of $\left[A_{0}\right]$ and $\left[B_{0}\right]$, the method can be easily extended to analyze systems that are undergoing large changes. Specifically, assume the modifications introduced in the perturbation matrices $[\delta A]$ and $[\delta B]$ are large. A simple iterative procedure is proposed whereby the perturbations are accounted for incrementally. The proposed iteration scheme is applicable to either a first-order or a second-order analysis. The proposed iterative procedure is as follows:

(1) Solve for the eigensolutions, $\lambda_{0 j}, \mathbf{x}_{0 j}$, and $\mathbf{y}_{0 j}$, of the nominal system whose matrices are given by $\left[A_{0}\right]$ and $\left[B_{0}\right]$.

(2) Divide the large perturbation matrices into $N$ smaller increments $[\delta A] / N$ and $[\delta B] / N$, where $N$ is chosen such that $|\delta A(i, j)| / N$ and $|\delta B(i, j)| / N$ are less than $10 \%$ of $\left|A_{0}(i, j)\right|$ and $\left|B_{0}(i, j)\right|$, respectively.

(3) Obtain the perturbed eigensolutions of the modified system whose matrices are given by $\left[A^{\prime}\right]=\left[A_{0}\right]+$ $[\delta A] / N$ and $\left[B^{\prime}\right]=\left[B_{0}\right]+[\delta B] / N$ using Equation (36) or Equation (37), depending on if a first-order or a second-order analysis is desired. Denote the perturbed eigenvalues as $\lambda_{j}^{\prime}$ and the perturbed right and left eigenvectors as $\mathbf{x}_{j}^{\prime}$ and $\mathbf{y}_{j}^{\prime}$, respectively.

(4) Assemble the perturbed right and left modal matrices $\left[X_{\text {pert }}\right]$ and $\left[Y_{\text {pert }}\right]$, whose columns correspond to $\mathbf{x}_{j}^{\prime}$ and $\mathbf{y}_{j}^{\prime}$, for $j=1, \ldots, p$.

(5) Normalize the perturbed modal matrices $\left[X_{\text {pert }}\right]$ and $\left[Y_{\text {pert }}\right]$ such that the diagonal elements of $\left[Y_{\text {pert }}\right]^{T}\left[A^{\prime}\right]\left[X_{\text {pert }}\right]$ equal 1.

(6) For the next iteration, let the nominal system matrices be $\left[A_{0}\right]=\left[A^{\prime}\right]$ and $\left[B_{0}\right]=\left[B^{\prime}\right]$, and let the nominal eigensolutions be $\lambda_{0 j}=\lambda_{j}^{\prime}, \mathbf{x}_{0 j}=\mathbf{x}_{j}^{\prime}$, and $\mathbf{y}_{0 j}=\mathbf{y}_{j}^{\prime}$.

(7) Repeat Steps 3 to 6 until $\left[A^{\prime}\right]=[A]$ and $\left[B^{\prime}\right]=[B]$.
Using the proposed scheme, the error between the exact and perturbed eigensolutions at each iteration becomes negligible if $N$ is sufficiently large. The perturbed eigensolutions are obtained iteratively until the smaller perturbations have accumulated to equal to the total modifications. It is also important to note that after each iteration, the perturbed eigenvectors must be properly normalized such that the diagonal elements of $\left[Y_{\text {pert }}\right]^{T}\left[A^{\prime}\right]\left[X_{\text {pert }}\right]$ are equal to 1 . This step is necessary because the perturbed eigenvectors must satisfy Equation (3).

Of great interest is also the accuracy of the perturbed eigenvectors, which can be determined by checking their self-compatibility using the orthogonality conditions. For the generalized eigenvalue problems, the following orthogonality check may be used:

$$
\left[I^{\prime}\right]=\left[Y_{\text {pert }}\right]^{T}[A]\left[X_{\text {pert }}\right]
$$

where $\left[I^{\prime}\right]$ denotes an orthogonal matrix, $[A]$ is the system matrix, and $\left[X_{\text {pert }}\right]$ and $\left[Y_{\text {pert }}\right]$ are normalized such that the diagonal elements of $\left[I^{\prime}\right]$ are identical ones. For an $i$ th-order analysis, the column vectors of the perturbed modal matrices $\left[X_{\text {pert }}\right]$ and $\left[Y_{\text {pert }}\right]$ are the $i$ th-order perturbed eigenvectors. Theoretically, if the perturbed modal matrices are exact, then the orthogonal matrix corresponds to the identity matrix. Since the perturbed modal matrices are approximate, the magnitudes of the nonzero off-diagonal terms of the orthogonal matrix can be used to infer the accuracy of the perturbed eigenvectors. As such, an error parameter for the modal matrices is defined, which is given by the average of the magnitudes of the off-diagonal terms, as follows:

$$
\delta=\frac{1}{p(p-1)} \sum_{i=1}^{p} \sum_{j=1, j \neq i}^{p}\left|I^{\prime}(i, j)\right|,
$$

where $p$ represents the size of the system matrices and $\left|I^{\prime}(i, j)\right|$ denotes the absolute value of the $(i, j)$ th element of the orthogonal matrix $\left[I^{\prime}\right]$. The smaller the value of $\delta$ is, the more accurate the perturbed modal matrices are.

2.3. Triple Product Method. After the completion of the straightforward perturbation method or the incremental perturbation method, the following triple product is calculated:

$$
\left[Y_{\text {pert }}\right]^{T}[B]\left[X_{\text {pert }}\right] \text {, }
$$

assuming these perturbed modal matrices have been properly normalized so that the diagonal elements of Equation (38) are all ones. If the perturbed modal matrices were replaced with the exact modal matrices, then the diagonal elements of Equation (40) correspond to the exact eigenvalues and all the off-diagonal terms will be identically zero. Since the perturbation analysis is only an approximation, the diagonal terms from the triple product can also be used to approximate the eigenvalues. In evaluating Equation (40), the modal matrices can be obtained via either a first-order or secondorder analysis. Interestingly, numerical experiments show that using an ith-order perturbation analysis, the diagonal elements of Equation (40) are significantly more accurate than those obtained using the ith-order straightforward 
perturbation method. This drastic improvement in accuracy is achieved by performing one additional step consisting of simple matrix multiplication. As such, in all of the examples, the diagonal elements of the triple product of Equation (40) will also be presented, both to validate the accuracy of the perturbed eigenvectors and to offer yet another approximation for the eigenvalues of the modified system.

\section{Results}

Three different methods are proposed to obtain the eigencharacteristics of modified symmetric or asymmetric systems. They consist of the straightforward perturbation method, the incremental perturbation method, and the triple product method. Each of these methods can be applied using either a first-order or second-order perturbation analysis. Using the straightforward perturbation method, the approximate eigensolutions are readily obtained by the direct application of Equations (33)-(35). Using the incremental perturbation method, Equations (33)-(35) are still applicable, except the perturbations are divided into $N$ increments, and the approximate eigencharacteristics of the modified system are obtained iteratively. Lastly, using the triple product method, the accuracy of the approximate eigenvalues given by the diagonal elements of Equation (40) becomes dramatically improved at the cost of only one additional step consisting of simple matrix multiplication. In the following, the eigensolutions for various symmetric and asymmetric systems will be obtained using the methods presented in this paper. For brevity, only the governing equations for the unperturbed system will be presented in each example. The governing equations for the perturbed system are identical to those of the unperturbed system, except that the perturbed system matrices will include the structural modifications that are subsequently introduced.

3.1. Torque-Free Rigid Body. Consider a torque-free rigid body as shown in Figure 1 [5], which is symmetric about

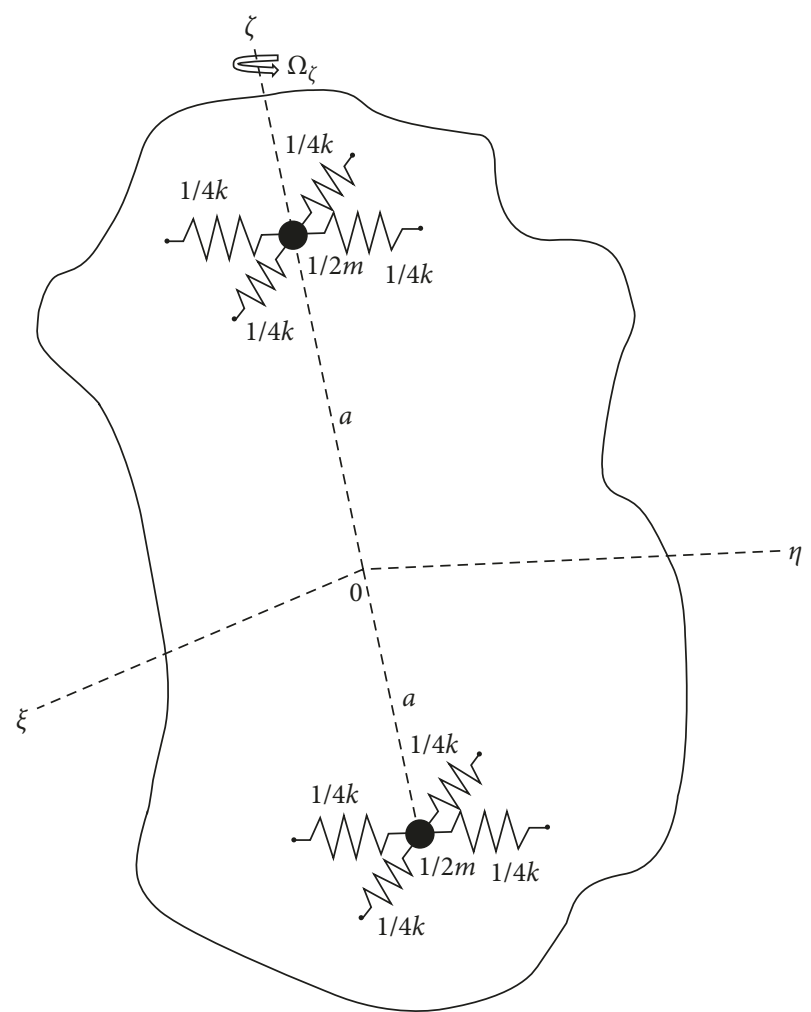

Figure 1: A torque-free rigid body.

axis $\zeta$. Two masses, each $m / 2$, are located at distances $\zeta=$ $\pm a$ from the fixed center $O$. Each mass is supported by 4 springs, each with stiffness $k / 4$. The rigid body spins about axis $\zeta$ with a constant angular velocity of $\Omega_{\zeta}$. The mass moments of inertia of the rigid body about axes $\xi$, $\eta$, and $\zeta$ are $\alpha, \beta=\alpha$, and $c$, respectively. The mass moment of inertia of the entire body about a transverse axis is given by $\alpha^{\prime}=\alpha+m a^{2}$. The system's unperturbed matrices are given by [5]

$$
\begin{aligned}
{\left[A_{0}\right] } & =\left[\begin{array}{ccc|ccc}
m_{0} \omega_{0}^{2} & 0 & 0 & 0 & 0 & 0 \\
0 & m_{0} \omega_{0}^{2} & 0 & 0 & 0 & 0 \\
0 & 0 & m_{0} & 0 & 0 & m_{0} a_{0} \\
\hline 0 & 0 & 0 & m_{0} & -m_{0} a_{0} & 0 \\
0 & 0 & 0 & -m_{0} a_{0} & \alpha_{0}^{\prime} & 0 \\
0 & 0 & m_{0} a_{0} & 0 & 0 & \alpha_{0}^{\prime}
\end{array}\right] \\
{\left[B_{0}\right] } & =\left[\begin{array}{ccc|ccc}
0 & -m_{0} \omega_{0}^{2} \Omega_{0} & -m_{0} \omega_{0}^{2} & 0 & 0 & 0 \\
m_{0} \omega_{0}^{2} \Omega_{0} & 0 & 0 & -m_{0} \omega_{0}^{2} & 0 & 0 \\
m_{0} \omega_{0}^{2} & 0 & 0 & -m_{0} \Omega_{0} & m_{0} a_{0} \Omega_{0} & 0 \\
\hline 0 & m_{0} \omega_{0}^{2} & m_{0} \Omega_{0} & 0 & 0 & m_{0} a_{0} \Omega_{0} \\
0 & 0 & -m_{0} a_{0} \Omega_{0} & 0 & 0 & \left(c_{0}-\alpha_{0}^{\prime}\right) \Omega_{0} \\
0 & 0 & 0 & -m_{0} a_{0} \Omega_{0} & -\left(c_{0}-\alpha_{0}^{\prime}\right) \Omega_{0} & 0
\end{array}\right]
\end{aligned}
$$


where $\alpha_{0}^{\prime}=\alpha_{0}+m_{0} a_{0}^{2}$. For definiteness, the original and unperturbed system's parameters are $\Omega_{\zeta}=\Omega_{0}=5 \times 10^{1} \mathrm{rad} / \mathrm{s}$, $\omega_{0}=6 \times 10^{1} \mathrm{rad} / \mathrm{s}, \quad c_{0} / \alpha_{0}=1.5, a_{0}=2 \mathrm{~m}, m_{0}=2.5 \times 10^{-2} \mathrm{~kg}$, $k_{0}=9 \times 10^{1} \mathrm{~N} / \mathrm{m}$, and $\alpha_{0}=1 \mathrm{~kg} \cdot \mathrm{m}^{2}$.

After an initial analysis has been performed, design changes are introduced. Let $x$ denote an arbitrary system parameter. Assume it is perturbed systematically from its initial value of $x_{0}$ as follows:

$$
x=x_{0}(1+\sigma \delta x)
$$

where $\delta x$ is arbitrarily chosen from a set of random numbers with zero mean and a standard deviation of one, and $\sigma$ represents a disorder strength that can be varied. Together, $\sigma \delta x$ denotes the percentage change in $x_{0}$. Thus, a large $\sigma$ value implies a large perturbation from the nominal value. All the modified system parameters are perturbed in this manner.

For the torque-free rigid body, the natural frequencies $\omega$ are the imaginary part of the eigenvalues $\lambda$, i.e., $\omega=\operatorname{imag}(\lambda)$. Suppose the modified parameters $m$ and $a$ deviate from their nominal values such that $m=m_{0}(1+\sigma \delta m)$ and $a=a_{0}(1+\sigma \delta a), \quad$ where $\quad \delta m=-4.4070 \times 10^{-1} \quad$ and $\delta a=8.4650 \times 10^{-1}$. Figure 2 shows the natural frequencies as a function of $\sigma$. These natural frequencies are obtained via five different means: by solving the problem exactly, by using the straightforward perturbation method via a first-order or a second-order analysis, and using the triple product method of Equation (40), where the modal matrices consist of the first-order or the second-order perturbed eigenvectors. When $\sigma=0$, the system is unperturbed. Note that for small values of $\sigma$, all the approximate natural frequencies track the exact perturbed natural frequencies accurately. As the magnitude of $\sigma$ increases, while the first-order and second-order straightforward perturbation approximations deviate from the exact values, the approximations obtained from the diagonal elements of the triple product, using either the first-order or second-order perturbed modal matrices, are indistinguishable from the exact natural frequencies. The fact that the diagonal elements track the exact natural frequencies so closely also validates the accuracy of the perturbed matrices. Table 1 tabulates these natural frequencies for $\sigma=0.65$. Thus, $m$ and $a$ deviate from their nominal values by approximately $\sigma \delta m \approx-28.6 \%$ and $\sigma \delta a \approx 55.0 \%$, respectively. The numerical value in the parentheses denotes the percentage error in the natural frequencies, defined as

$$
\varepsilon=\frac{\mid \text { exact }- \text { approximate } \mid}{\mid \text { exact } \mid} \times 100 \% \text {. }
$$

Note the drastic improvement in the approximate natural frequencies obtained using a first-order triple product approach compared with using a first-order straightforward expansion, especially for the fundamental natural frequency. This significant improvement in accuracy is the result of carrying out another step consisting of a simple matrix multiplication given by Equation
(40). Interestingly, the approximate natural frequencies obtained using a first-order triple product approach are even more accurate that those obtained using a secondorder straightforward perturbation method. Note that among the different methods, the error parameters using the triple product with the second-order modal matrices are the smallest.

If the error parameter $\varepsilon$ is deemed too large for a disorder strength of $\sigma=0.65$ or if a larger value of $\sigma$ is applied, which would represent larger perturbations in the system, dividing the perturbations into smaller pieces and computing the approximations through an incremental method can be utilized. Although this incremental procedure involves more computation, it is deemed worthwhile when dealing with large perturbations in order to obtain accurate approximations. Consider now the case where $\sigma=1.8$. Figure 3 shows the natural frequencies obtained using the five methods mentioned previously as a function of $N$. These approximate natural frequencies monotonically converge to their respective exact values though some methods converge much more quickly than others. Note that the approximate fundamental natural frequency converges to the exact $\omega_{1}$ from above, while the other approximate natural frequencies converge to their respective exact values from below. By $N=5$, the triple product method with the first-order and second-order modal matrices leads to approximations that are nearly exact while the first-order incremental perturbation method is still very inaccurate by $N=10$. Across all values of $N$, the results of the triple product approximations via a second-order analysis are the most accurate. Table 2 illustrates the numerical results of Figure 3 for $N=8$. With eight iterations, the perturbations at each iteration are less than $10 \%$ of the original system matrices. At $N=8$, the errors for all three natural frequencies are reduced by at least an order of magnitude when approximating the new natural frequencies using the diagonal elements of the second-order triple product as opposed to simply the incremental perturbation method via a first-order analysis. With the second-order triple product, the final error percentages of all three natural frequencies are below $0.83 \%$, substantially smaller than the percentage deviations in $m_{0}$ and $a_{0}$ that are introduced into the system, which are approximately $\sigma \delta m \approx-79.3 \%$ and $\sigma \delta a \approx 152.3 \%$, respectively. To assess the accuracy of the perturbed modal matrices for this torque-free rigid body system, consider the error parameter given by Equation (39), where $p=6$. Figure 4 shows $\delta$ as a function of $N$ for $\sigma=1.8$, obtained using a first-order or a second-order incremental perturbation analysis. Note that $\delta$ decreases with $N$ and that the error parameter for the second-order modal matrices decreases more quickly than the error parameter for the firstorder modal matrices.

3.2. Two-Degrees-of-Freedom Gyroscopic System. Figure 5 shows a two-degrees-of-freedom gyroscopic system 


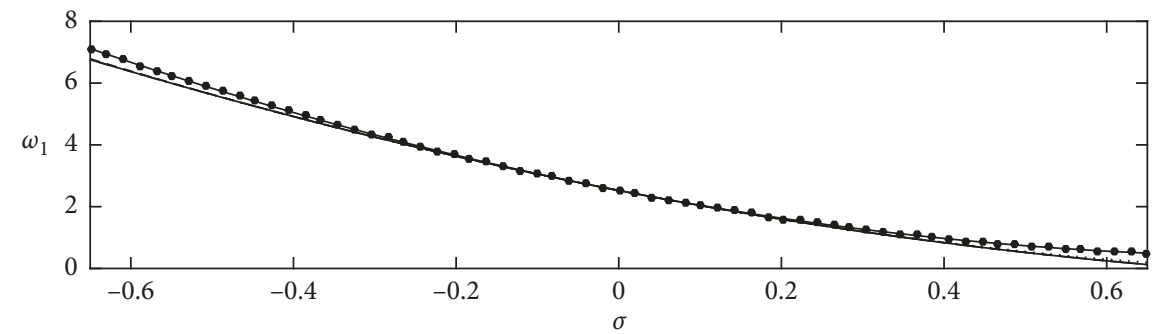

(a)

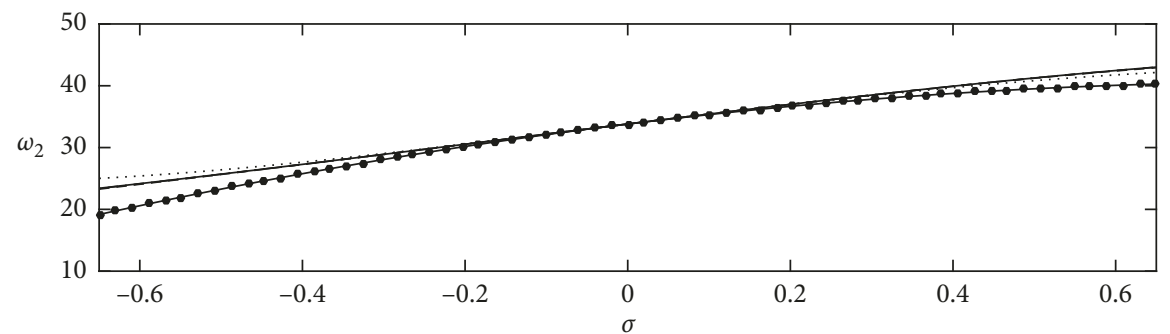

(b)

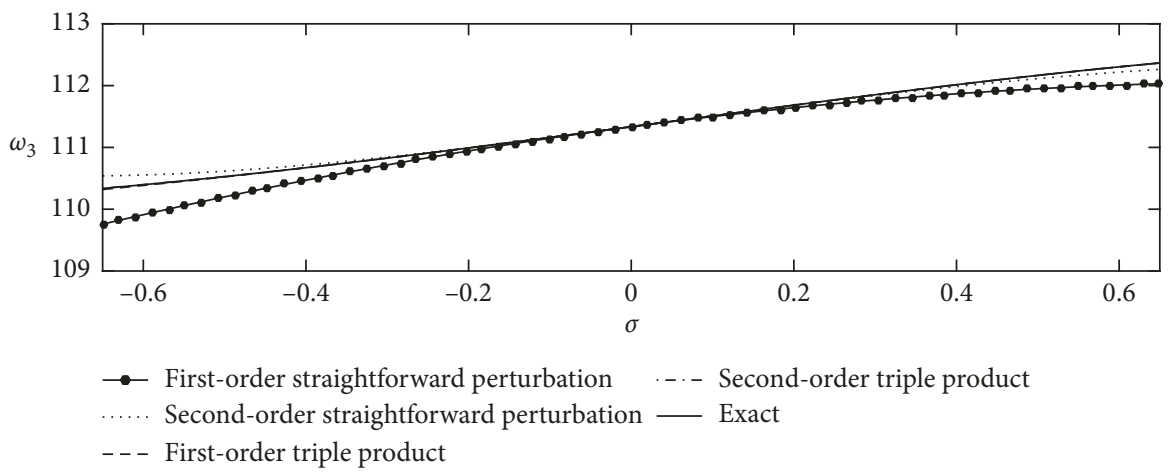

(c)

FIgURE 2: The exact and approximate natural frequencies ( $\mathrm{rad} / \mathrm{s})$ of the torque-free rigid body as a function of $\sigma$.

TABLe 1: The exact and approximate natural frequencies for the perturbed torque-free rigid body of Figure 1 for $\sigma=0.65$.

\begin{tabular}{cccccccc}
\hline & Exact & $\begin{array}{c}\text { First-order straightforward } \\
\text { perturbation }\end{array}$ & $\begin{array}{c}\text { Second-order straightforward } \\
\text { perturbation }\end{array}$ & First-order triple product & Second-order triple product \\
\hline$\omega_{1}$ & $1.2592 \times 10^{-1}$ & $4.9493 \times 10^{-1}\left(2.93 \times 10^{2}\right)$ & $1.9843 \times 10^{-1}\left(5.75 \times 10^{1}\right)$ & $1.2801 \times 10^{-1}\left(1.66 \times 10^{0}\right)$ & $1.2503 \times 10^{-1}$ & $\left(7.03 \times 10^{-1}\right)$ \\
$\omega_{2}$ & $4.3014 \times 10^{1}$ & $4.0276 \times 10^{1}$ & $\left(6.36 \times 10^{0}\right)$ & $4.2147 \times 10^{1}\left(2.01 \times 10^{0}\right)$ & $4.2925 \times 10^{1}\left(2.08 \times 10^{-1}\right)$ & $4.3004 \times 10^{1}$ & $\left(2.43 \times 10^{-2}\right)$ \\
$\omega_{3}$ & $1.1236 \times 10^{2}$ & $1.1203 \times 10^{2}$ & $\left(2.99 \times 10^{-1}\right)$ & $1.1226 \times 10^{2}\left(9.32 \times 10^{-2}\right)$ & $1.1236 \times 10^{2}\left(1.98 \times 10^{-3}\right)$ & $1.1236 \times 10^{2}$ & $\left(5.69 \times 10^{-4}\right)$ \\
\hline
\end{tabular}

analyzed by Meirovitch and Ryland [10]. The governing equations for the unperturbed system are given by

$$
\left[M_{0}\right] \ddot{\mathbf{q}}_{0}+\left(\left[C_{0}\right]+\left[G_{0}\right]\right) \dot{\mathbf{q}}_{0}+\left[K_{0}\right] \mathbf{q}_{0}=0,
$$

where an overdot denotes a time derivative, $\left[M_{0}\right],\left[C_{0}\right]$, and $\left[K_{0}\right]$ are the mass, damping, and stiffness matrices of the nominal system, respectively, $\left[G_{0}\right]$ denotes the unperturbed gyroscopic damping matrix, and $\mathbf{q}_{0}$ is the vector of generalized coordinates for the nominal system. The system matrices are all of size $2 \times 2$, and $\mathbf{q}_{0}$ is of length 2 . The nominal system matrices are

$$
\begin{aligned}
{\left[M_{0}\right] } & =\left[\begin{array}{cc}
m_{0} & 0 \\
0 & m_{0}
\end{array}\right], \\
{\left[G_{0}\right] } & =\left[\begin{array}{cc}
0 & -2 m_{0} \Omega_{0} \\
2 m_{0} \Omega_{0} & 0
\end{array}\right], \\
{\left[C_{0}\right] } & =\left[\begin{array}{cc}
c_{0} & 0 \\
0 & 0
\end{array}\right], \\
{\left[K_{0}\right] } & =\left[\begin{array}{cc}
k_{01}-m_{0} \Omega_{0}^{2} & 0 \\
0 & k_{02}-m_{0} \Omega_{0}^{2}
\end{array}\right] .
\end{aligned}
$$




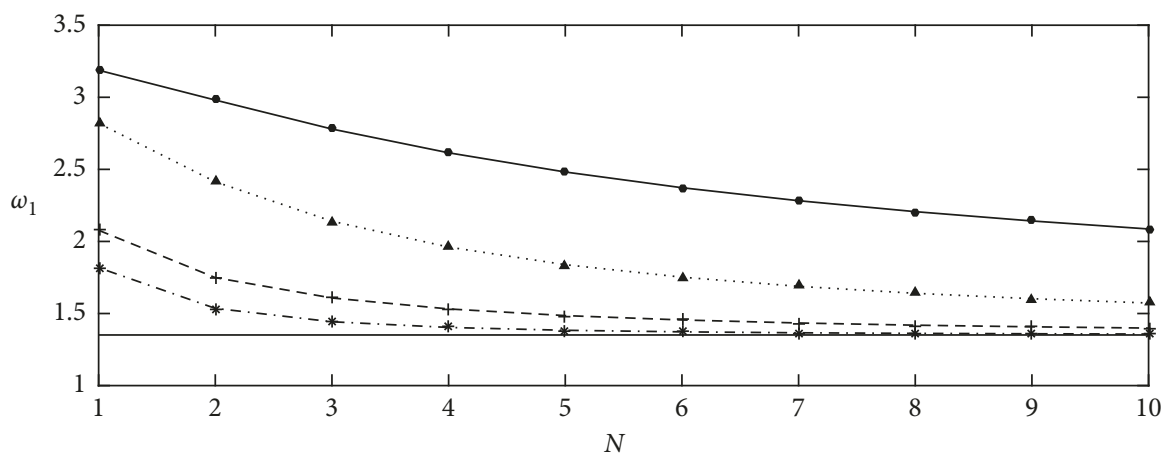

(a)

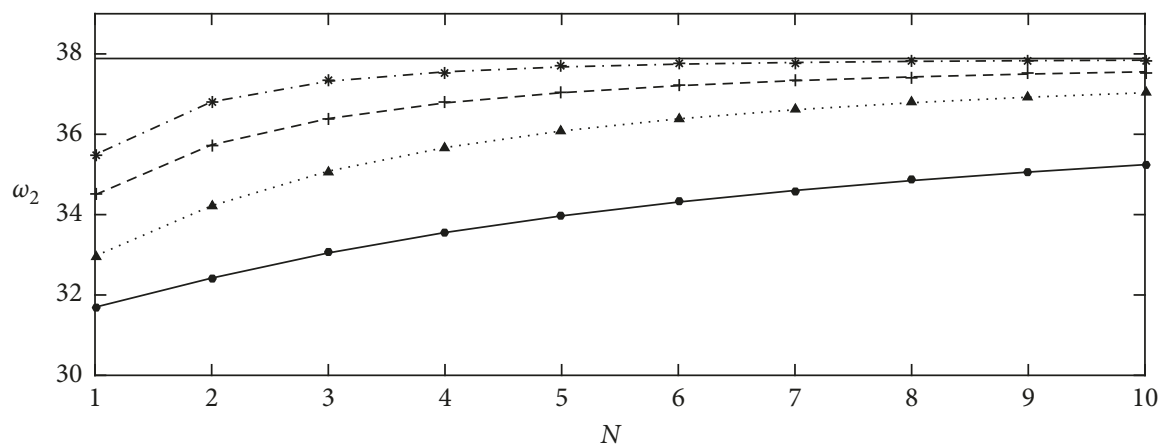

(b)

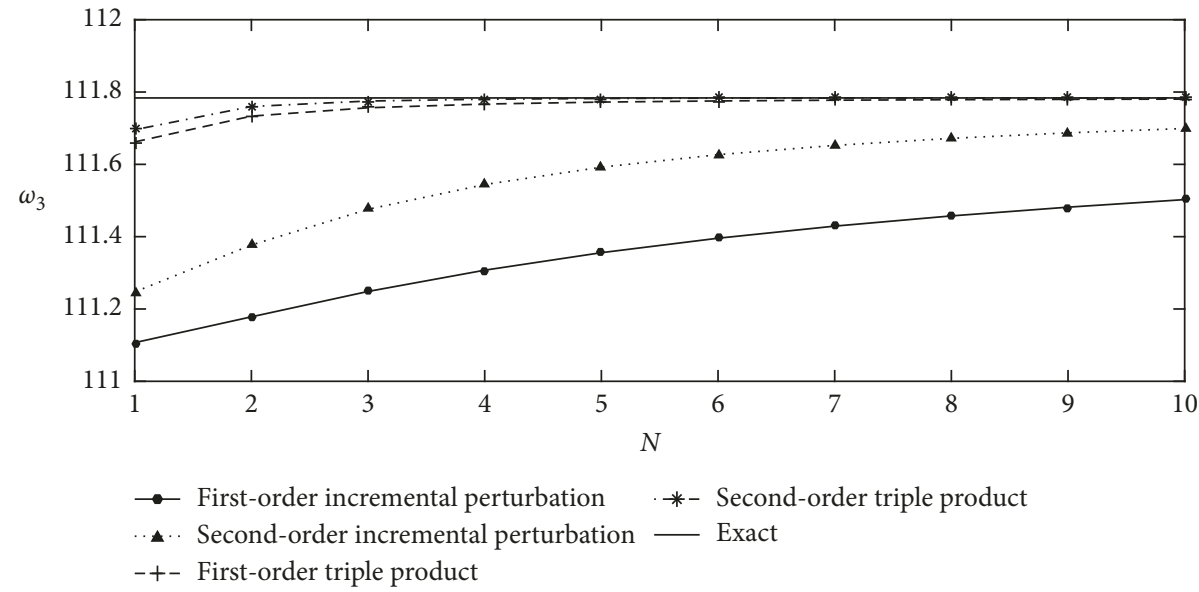

(c)

Figure 3: The approximate natural frequencies of the torque-free rigid body as a function of $N$ for $\sigma=1.8$.

TABLE 2: The exact and approximate natural frequencies for the perturbed torque-free rigid body at $N=8$ for $\sigma=1.8$.

\begin{tabular}{cccccc}
\hline & Exact & $\begin{array}{c}\text { First-order incremental } \\
\text { perturbation }\end{array}$ & $\begin{array}{c}\text { Second-order incremental } \\
\text { perturbation }\end{array}$ & $\begin{array}{c}\text { First-order } \\
\text { triple product }\end{array}$ & $\begin{array}{c}\text { Second-order } \\
\text { triple product }\end{array}$ \\
\hline$\omega_{1}$ & $1.3511 \times 10^{0}$ & $2.2065 \times 10^{0}\left(6.33 \times 10^{1}\right)$ & $1.6401 \times 10^{0}\left(2.13 \times 10^{1}\right)$ & $1.4190 \times 10^{0}\left(5.02 \times 10^{0}\right)$ & $1.3622 \times 10^{0}\left(8.22 \times 10^{-1}\right)$ \\
$\omega_{2}$ & $3.7885 \times 10^{1}$ & $3.4847 \times 10^{1}\left(8.01 \times 10^{0}\right)$ & $3.6785 \times 10^{1}\left(2.90 \times 10^{0}\right)$ & $3.7427 \times 10^{1}\left(1.20 \times 10^{0}\right)$ & $3.7813 \times 10^{1}\left(1.89 \times 10^{-1}\right)$ \\
$\omega_{3}$ & $1.1178 \times 10^{2}$ & $1.1145 \times 10^{2}\left(2.91 \times 10^{-1}\right)$ & $1.1167 \times 10^{2}\left(9.99 \times 10^{-2}\right)$ & $1.1177 \times 10^{2}\left(4.38 \times 10^{-3}\right)$ & $1.1178 \times 10^{2}\left(1.84 \times 10^{-5}\right)$ \\
\hline
\end{tabular}

To solve for the eigensolutions of the system shown in Equation (44), the state vector approach [28] is used. Introducing a state vector of length 4 of the form

$$
\mathbf{x}_{0}=\left[\begin{array}{c}
\dot{\mathbf{q}}_{0} \\
\mathbf{q}_{0}
\end{array}\right],
$$

allows Equation (44) to be rewritten as 


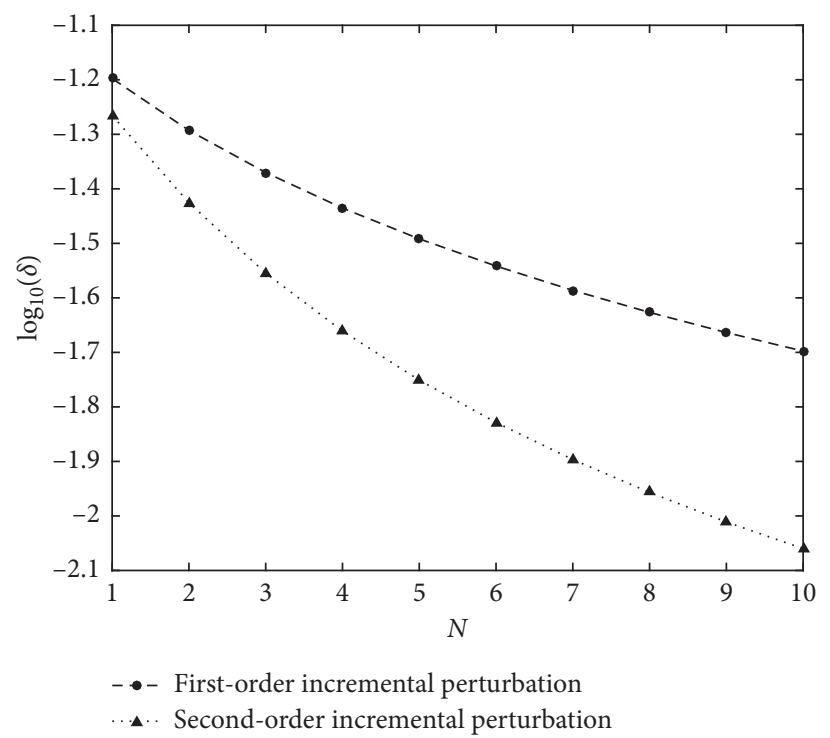

FiguRE 4: The error parameter, $\delta$, for the perturbed modal matrices of the torque-free rigid body as a function of $N$ for $\sigma=1.8$.

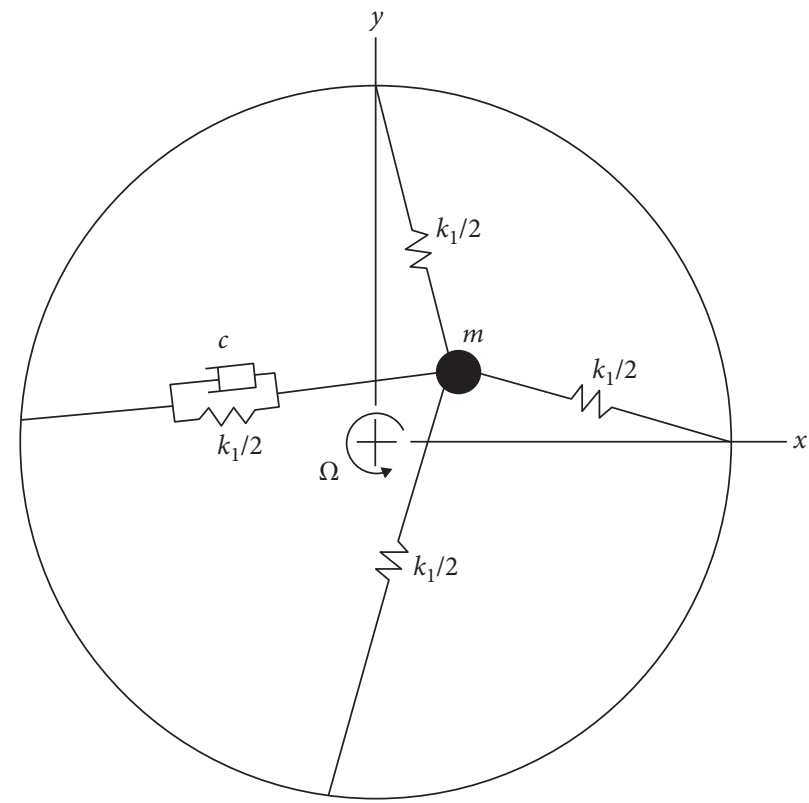

FIgURe 5: A two-degree-of-freedom gyroscopic system.

$$
\left[A_{0}\right] \dot{\mathbf{x}}_{0}-\left[B_{0}\right] \mathbf{x}_{0}=0,
$$

where $\left[A_{0}\right]$ and $\left[B_{0}\right]$ are the nominal state matrices of size $4 \times 4$ given by

$$
\begin{aligned}
& {\left[A_{0}\right]=\left[\begin{array}{cc}
{\left[M_{0}\right]} & {[0]} \\
{[0]} & {\left[K_{0}\right]}
\end{array}\right],} \\
& {\left[B_{0}\right]=\left[\begin{array}{cc}
-\left[C_{0}\right]-\left[G_{0}\right] & {\left[-K_{0}\right]} \\
{\left[K_{0}\right]} & {[0]}
\end{array}\right] .}
\end{aligned}
$$

Figure 5 is an example of a damped, asymmetric system due to the presence of the gyroscopic damping matrix $\left[G_{0}\right]$.
For definiteness, the original or unperturbed system parameters are $m_{0}=1 \mathrm{~kg}, \Omega_{0}=1 \mathrm{rad} / \mathrm{s}, \quad c_{0}=1 \times 10^{-1} \mathrm{~N} \cdot \mathrm{s} / \mathrm{m}$, $k_{01}=3 \mathrm{~N} / \mathrm{m}$, and $k_{02}=4 \mathrm{~N} / \mathrm{m}$. To illustrate that the perturbation methods presented in this paper can also be used when an asymmetric damping matrix is perturbed, the parameters $c_{0}$ and $m_{0}$ are subjected to modifications; thus $c=c_{0}(1+\sigma \delta c)$ and $m=m_{0}(1+\sigma \delta m)$. The random numbers used for the perturbations are $\delta c=-7.2540 \times 10^{-1}$ and $\delta m=2.8480 \times 10^{-1}$. Figures 6 and 7 illustrate the real and imaginary parts of the system's eigenvalues (obtained exactly, with the straightforward perturbation method via a first-order or a second-order analysis, and with the diagonal elements of Equation (40) using the first-order or 


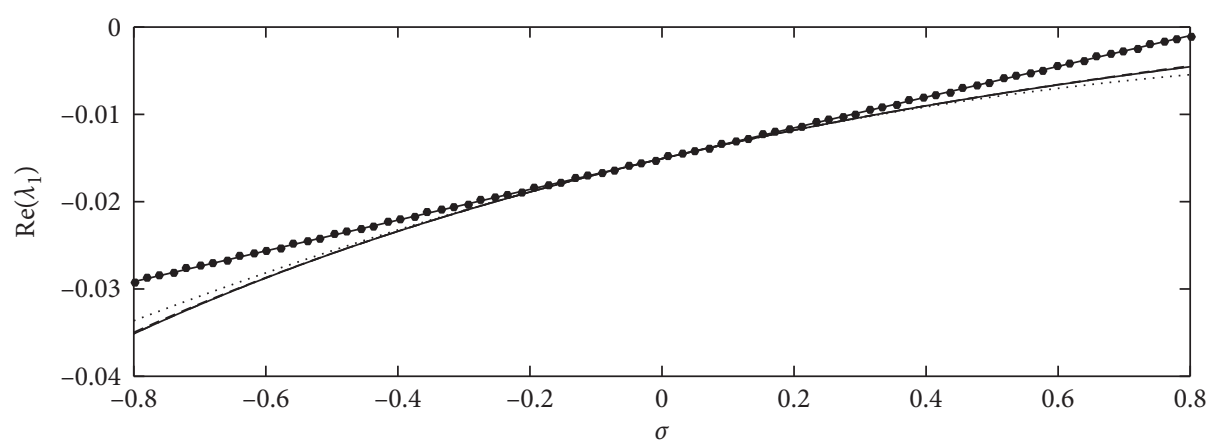

(a)

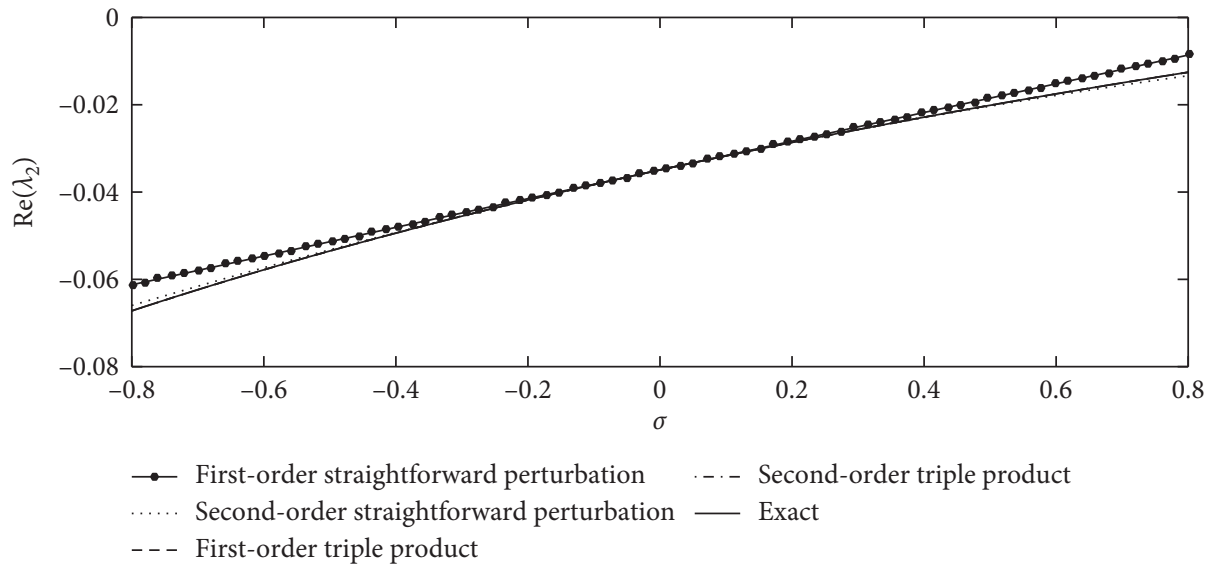

(b)

Figure 6: The real parts of the exact and approximate eigenvalues of the two-degree-of-freedom gyroscopic system as a function of $\sigma$.

second-order perturbed modal matrices) as a function of $\sigma$. Note the diagonal elements of both triple products almost lie on top of the exact eigenvalues, which validates the accuracy of the perturbed modal matrices, and they are more accurate than the second-order straightforward perturbation approximations over the entire range of $\sigma$. Tables 3 and 4 compare the real and imaginary parts of the exact and approximate natural frequencies for $\sigma=0.8$. Note again that the first-order triple product method yields approximate eigenvalues that are more accurate than those obtained using a second-order straightforward perturbation analysis. Moreover, note that using the second-order triple product method, the error parameters for both the real and imaginary parts of the eigenvalues are reduced by at least two orders of magnitude compared to those when using the firstorder straightforward perturbation method. Additionally, for $\sigma=0.8$, the real parts of the eigenvalues obtained using the second-order triple product method have $\varepsilon$ that are all less than $0.16 \%$, and the imaginary parts have $\varepsilon$ all less than $0.0095 \%$. These errors are significantly smaller than the percentage changes in $c_{0}$ and $m_{0}$, which are approximately $\sigma \delta m \approx 22.7 \%$ and $\sigma \delta c \approx-58.0 \%$, respectively.

Assume larger perturbations are introduced to the system by using $\sigma=1.37$. Figures 8 and 9 show the eigenvalues found using the five methods mentioned previously as a function of $N$. Note that using a first-order incremental perturbation method, the real parts of the eigenvalue converge to their exact values from above, while the imaginary parts converge to their exact values from below. The trend is reversed using the second-order incremental perturbation analysis, i.e., the real and imaginary parts converge to their exact values from below and above, respectively. For the chosen set of structural parameters, the modified system is stable. However, Figure 8 shows that the first-order incremental perturbation method yields approximate eigenvalues that erroneously indicate the system is unstable. In the case, because the real components of the system's exact eigenvalues are close to 0 , it is important to use a sufficiently large value of $N$ to accurately capture the system's stability. Also, note that the approximate eigenvalues found using the diagonal elements of the first-order and second-order triple products converge rapidly to the exact eigenvalues for $N$ as small as 3 , and for $N=10$, the approximate and exact eigenvalues become indistinguishable, despite the large perturbations that are introduced in the system. Tables 5 and 6 show the corresponding numerical results. Note that $\varepsilon$ of the second-order triple product is at least four orders of magnitude smaller than $\varepsilon$ of the first-order incremental perturbation method. Figure 10 shows the error parameter $\delta$ for the first-order and second-order perturbed modal matrices, obtained using Equation (39) with $p=4$, as 


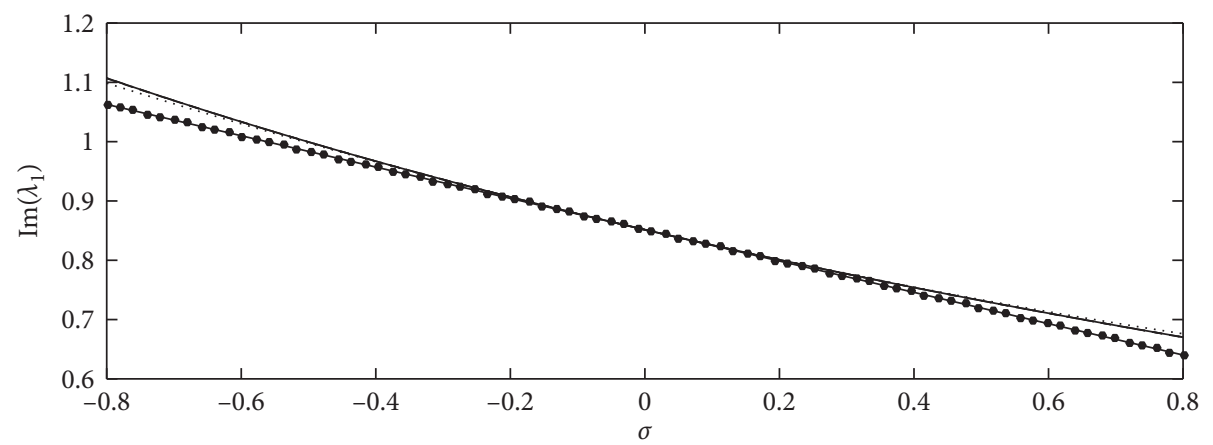

(a)

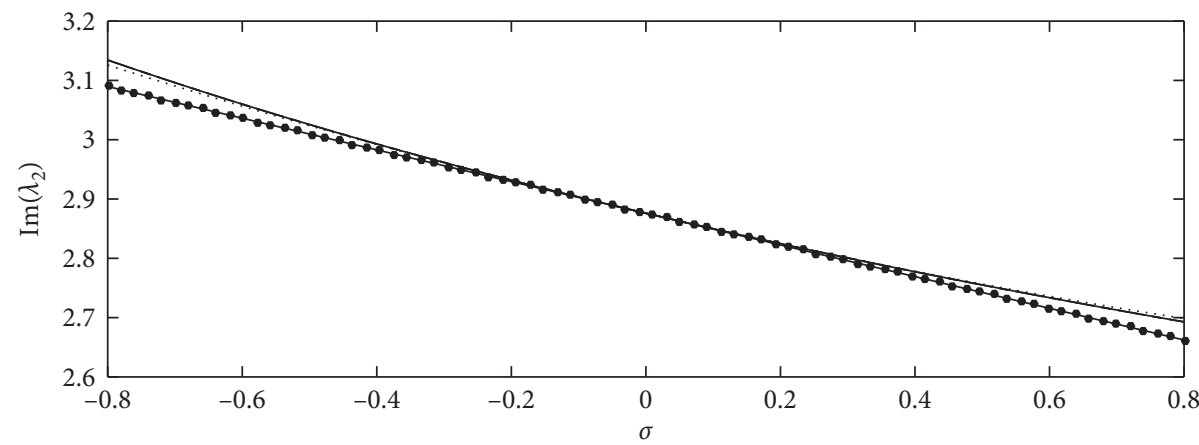

-. First-order straightforward perturbation $\ldots$-.. Second-order triple product
.... Second-order straightforward perturbation _ $\quad$ Exact
- - - First-order triple product

(b)

FIgURE 7: The imaginary parts of the exact and approximate eigenvalues of the two-degree-of-freedom gyroscopic system as a function of $\sigma$.

TABLE 3: The real parts of the exact and approximate natural frequencies for the perturbed two-degree-of-freedom gyroscopic system of Figure 5 for $\sigma=0.8$.

\begin{tabular}{|c|c|c|c|c|c|}
\hline & Exact & $\begin{array}{l}\text { First-order straightforward } \\
\text { perturbation }\end{array}$ & $\begin{array}{l}\text { Second-order straightforward } \\
\text { perturbation }\end{array}$ & First-order triple product & $\begin{array}{l}\text { Second-order triple } \\
\text { product }\end{array}$ \\
\hline $\operatorname{Re}\left(\lambda_{1}\right)$ & $-4.5435 \times 10^{-3}$ & $-9.7877 \times 10^{-4}\left(7.84 \times 10^{1}\right)$ & $-5.4541 \times 10^{-3}(2.00>$ & $\begin{aligned}-4.4447 \times 10^{-3} \\
\\
\left(2.17 \times 10^{0}\right)\end{aligned}$ & $\begin{array}{c}-4.5366 \times 10^{-3} \\
\left(1.50 \times 10^{-1}\right)\end{array}$ \\
\hline $\operatorname{Re}\left(\lambda_{2}\right)$ & $-1.2546 \times 10^{-2}$ & $-8.6132 \times 10^{-3}\left(3.13 \times 10^{1}\right)$ & $-1.3344 \times 10^{-2}\left(6.35 \times 10^{0}\right)$ & $\begin{array}{c}-1.2565 \times 10^{-2} \\
\left(1.53 \times 10^{-1}\right)\end{array}$ & $\begin{array}{c}-1.2540 \times 10^{-2} \\
\left(4.60 \times 10^{-2}\right)\end{array}$ \\
\hline
\end{tabular}

TABLE 4: The imaginary parts of the exact and approximate natural frequencies for the perturbed two-degree-of-freedom gyroscopic system of Figure 5 for $\sigma=0.8$.

\begin{tabular}{lccccc}
\hline & Exact & $\begin{array}{c}\text { First-order } \\
\text { straightforward } \\
\text { perturbation }\end{array}$ & $\begin{array}{c}\text { Second-order } \\
\text { straightforward } \\
\text { perturbation }\end{array}$ & First-order triple product & $\begin{array}{c}\text { Second-order triple } \\
\text { product }\end{array}$ \\
\hline $\operatorname{Im}\left(\lambda_{1}\right)$ & $6.7034 \times 10^{-1}$ & $6.4020 \times 10^{-1}\left(4.49 \times 10^{0}\right)$ & $6.7603 \times 10^{-1}\left(8.49 \times 10^{-1}\right)$ & $\begin{array}{c}6.7000 \times 10^{-1} \\
\left(5.01 \times 10^{-2}\right)\end{array}$ & $\begin{array}{c}6.7027 \times 10^{-1} \\
\left(9.43 \times 10^{-3}\right)\end{array}$ \\
$\operatorname{Im}\left(\lambda_{2}\right)$ & $2.6928 \times 10^{0}$ & $2.6623 \times 10^{0}\left(1.13 \times 10^{0}\right)$ & $2.6985 \times 10^{0}\left(2.11 \times 10^{-1}\right)$ & $2.6927 \times 10^{0}\left(2.90 \times 10^{-3}\right)$ & $2.6928 \times 10^{0}\left(3.95 \times 10^{-4}\right)$ \\
\hline
\end{tabular}

a function of $N$. It clearly shows a rapid decrease of $\delta$, which implies a high accuracy in the perturbed eigenvectors, especially those obtained using a second-order analysis.
3.3. Whirling Beam with Gyroscopic Damping. Figure 11 shows a whirling shaft with a lumped mass $M$ attached at its midspan [12]. The shaft's mass is uniformly distributed with mass per unit length $m$. The shaft has 


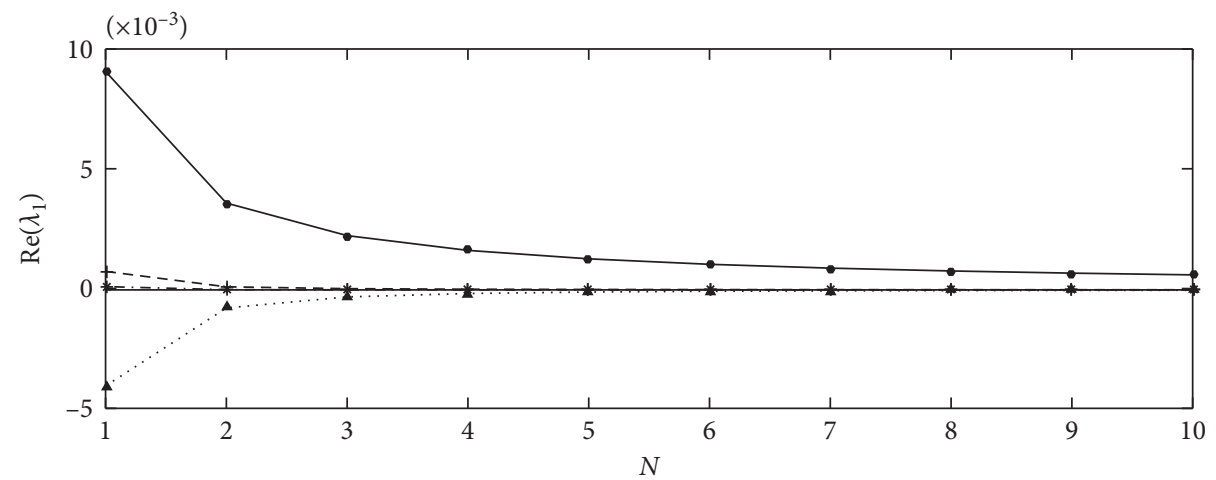

(a)

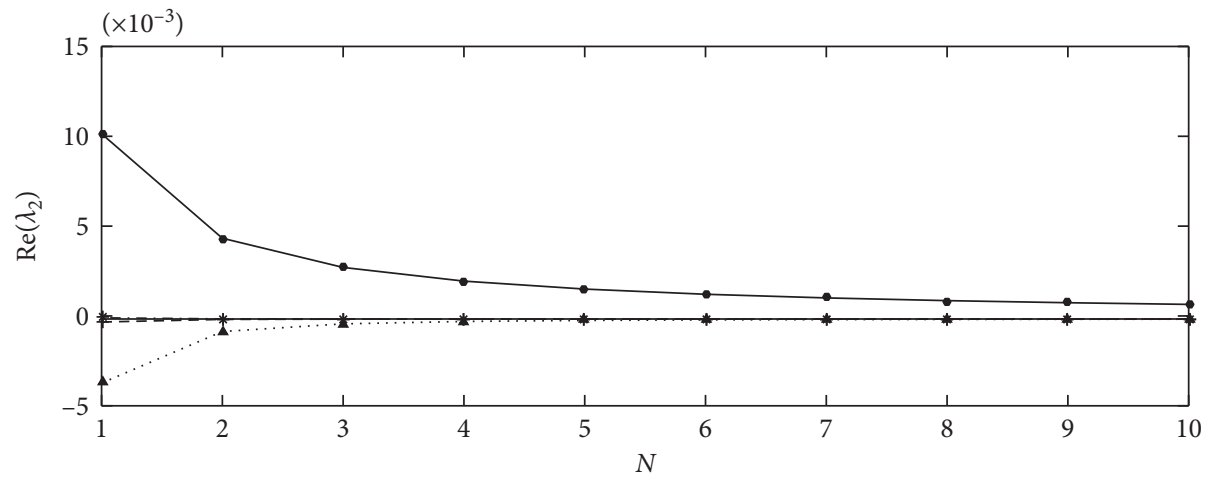

- First-order incremental perturbation

*- Second-order triple product

- - Second-order incremental perturbation

— Exact

-+- First-order triple product

(b)

FIGURE 8: The real parts of the approximate eigenvalues of the two-degree-of-freedom gyroscopic system as a function of $N$ for $\sigma=1.37$.

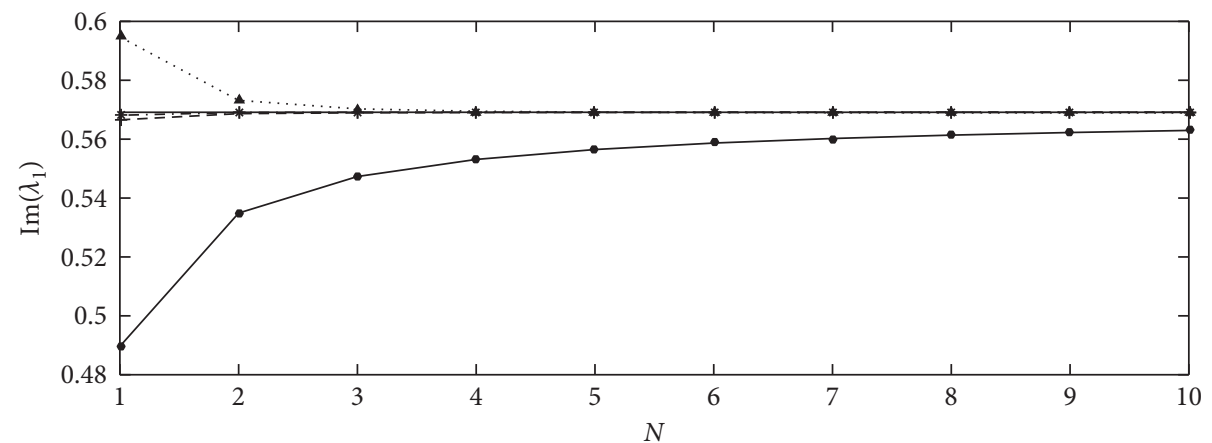

(a)

Figure 9: Continued. 


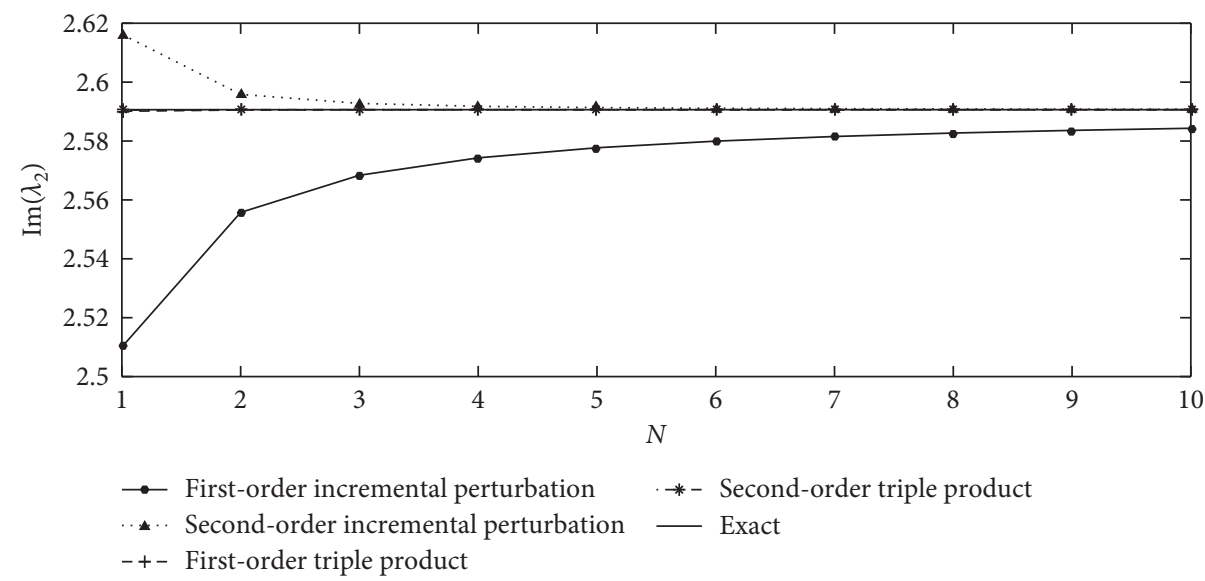

(b)

FIGURE 9: The imaginary parts of the approximate eigenvalues of the two-degree-of-freedom gyroscopic system as a function of $N$ for $\sigma=1.37$.

TABLE 5: The real parts of the exact and approximate natural frequencies for the perturbed two-degree-of-freedom gyroscopic system at $N=10$ for $\sigma=1.37$

\begin{tabular}{ccccc}
\hline Exact & $\begin{array}{c}\text { First-order incremental } \\
\text { perturbation }\end{array}$ & $\begin{array}{c}\text { Second-order incremental } \\
\text { perturbation }\end{array}$ & First-order triple product & Second-order triple product \\
\hline $\operatorname{Re}\left(\lambda_{1}\right)-5.4248 \times 10^{-5}$ & $\begin{array}{c}5.7165 \times 10^{-4} \\
\left(1.15 \times 10^{3}\right)\end{array}$ & $-7.0836 \times 10^{-5}\left(3.05 \times 10^{1}\right)$ & $-5.0406 \times 10^{-5}$ & $-5.4177 \times 10^{-5}\left(1.30 \times 10^{-1}\right)$ \\
$\operatorname{Re}\left(\lambda_{2}\right)-1.6881 \times 10^{-4}$ & $\begin{array}{c}6.4366 \times 10^{-4} \\
\left(4.81 \times 10^{2}\right)\end{array}$ & $-1.8005 \times 10^{-4}\left(6.65 \times 10^{0}\right)$ & $\left.-1.6942 \times 10^{0}\right)$ & \\
& & $\left(3.62 \times 10^{-1}\right)$ & $-1.6879 \times 10^{-4}\left(1.16 \times 10^{-2}\right)$ \\
\hline
\end{tabular}

TABLE 6: The imaginary parts of the exact and approximate natural frequencies for the perturbed two-degree-of-freedom gyroscopic system at $N=10$ for $\sigma=1.37$.

\begin{tabular}{cccccc}
\hline & Exact & $\begin{array}{c}\text { First-order incremental } \\
\text { perturbation }\end{array}$ & $\begin{array}{c}\text { Second-order incremental } \\
\text { perturbation }\end{array}$ & First-order triple product & Second-order triple product \\
\hline $\operatorname{Im}\left(\lambda_{1}\right)$ & $5.6913 \times 10^{-1}$ & $\begin{array}{c}5.6296 \times 10^{-1} \\
\left(1.08 \times 10^{0}\right)\end{array}$ & $\begin{array}{c}5.6989 \times 10^{-1} \\
\left(3.63 \times 10^{-2}\right)\end{array}$ & $5.6912 \times 10^{-1}$ & $5.6913 \times 10^{-1}$ \\
& & $\begin{array}{l}2.5843 \times 10^{0} \\
\left(2.43 \times 10^{-1}\right)\end{array}$ & $2.5907 \times 10^{0}\left(6.06 \times 10^{-3}\right)$ & $\left.2.5906 \times 10^{-5}\right)$ \\
$\operatorname{Im}\left(\lambda_{2}\right)$ & $2.5906 \times 10^{0}$ & $\left(1.08 \times 10^{-4}\right)$ & $2.5906 \times 10^{0}\left(1.59 \times 10^{-6}\right)$ \\
\hline
\end{tabular}

stiffnesses $\mathrm{EI}_{x}$ and $\mathrm{EI}_{y}$ about the $x$ and $y$ axis, respectively. The end supports provide bending moments proportional to the angular deflections, where the proportionality constants are $K_{1}$ at $Z=0$ and $K_{2}$ at $Z=L$. It is assumed that there is uniformly distributed external dissipation proportional by a constant $h$ to the absolute velocity, and an internal dissipation proportional by a constant $c$ to the velocity relative to the rotating axes. The equations of motion of the unperturbed system are given by [12]

$$
\left[M_{0}\right] \ddot{q}_{0}+\left(\left[C_{0}\right]+\left[G_{0}\right]\right) \dot{q}_{0}+\left(\left[K_{0}\right]+\left[H_{0}\right]\right) \mathbf{q}_{0}=0
$$

where the coefficient matrices in Equation (49) are partitioned into $s \times s$ submatrices

$$
\begin{aligned}
& {\left[M_{0}\right]=\left[\begin{array}{cc}
{\left[M^{s}\right]} & {[0]} \\
{[0]} & {\left[M^{s}\right]}
\end{array}\right],} \\
& {\left[G_{0}\right]=\left[\begin{array}{cc}
{[0]} & {\left[G^{s}\right]} \\
-\left[G^{s}\right] & {[0]}
\end{array}\right],} \\
& {\left[C_{0}\right]=\left[\begin{array}{cc}
{\left[C^{s}\right]} & {[0]} \\
{[0]} & {\left[C^{s}\right]}
\end{array}\right],} \\
& {\left[K_{0}\right]=\left[\begin{array}{cc}
{\left[K^{s 1}\right]} & {[0]} \\
{[0]} & {\left[K^{s 2}\right]}
\end{array}\right],} \\
& {\left[H_{0}\right]=\left[\begin{array}{cc}
{[0]} & {\left[H^{s}\right]} \\
-\left[H^{s}\right] & {[0]}
\end{array}\right] .}
\end{aligned}
$$

The $(i, j)$ th elements of the submatrices are given by [12] 


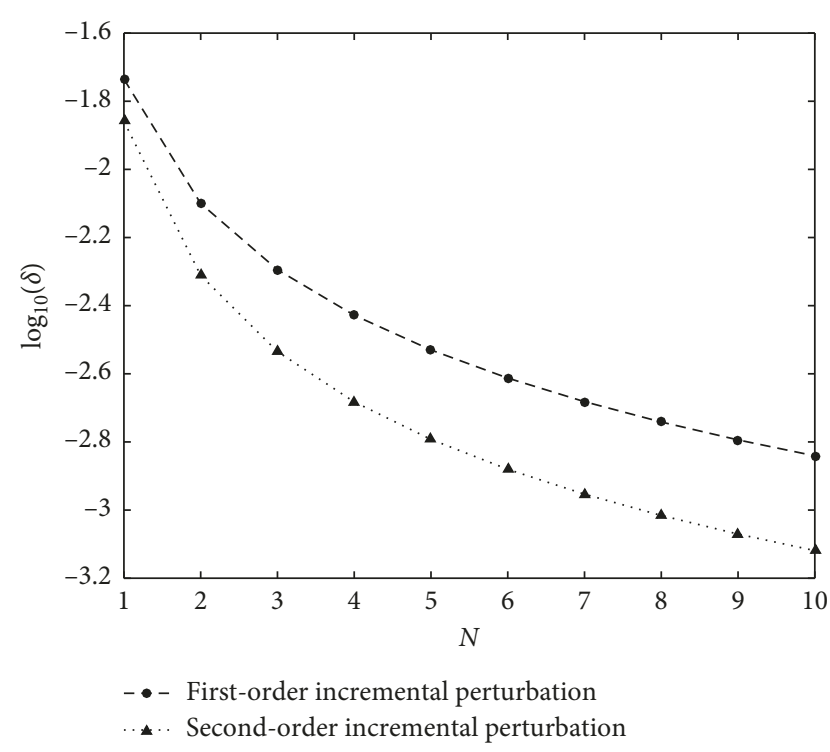

Figure 10: The error parameter, $\delta$, for the perturbed modal matrices of the two-degree-of-freedom gyroscopic system as a function of $N$ for $\sigma=1.37$.

$$
\begin{aligned}
M_{i j}^{s}= & m_{0} L_{0} \delta_{i}^{j}+2 M_{0} \sin \left(\frac{i \pi}{2}\right) \sin \left(\frac{j \pi}{2}\right), \\
G_{i j}^{s}= & -2 \Omega_{0} M_{i j}, \\
C_{i j}^{s}= & \left(c_{0}+h_{0}\right) L_{0} \delta_{i}^{j}, \\
K_{i j}^{s 1}= & 2\left(K_{01}+K_{02} \cos (i \pi) \cos (j \pi)\right)\left(\frac{i \pi}{L_{0}}\right)\left(\frac{j \pi}{L_{0}}\right) \\
& +\mathrm{EI}_{0 x}\left(\frac{i \pi}{L_{0}}\right)^{2}\left(\frac{j \pi}{L_{0}}\right)^{2} L_{0} \delta_{i}^{j}-\Omega_{0}^{2} M_{i j}, \\
K_{i j}^{s 2}= & 2\left(K_{01}+K_{02} \cos (i \pi) \cos (j \pi)\right)\left(\frac{i \pi}{L_{0}}\right)\left(\frac{j \pi}{L_{0}}\right) \\
& +\mathrm{EI}_{0 y}\left(\frac{i \pi}{L_{0}}\right)^{2}\left(\frac{j \pi}{L_{0}}\right)^{2} L_{0} \delta_{i}^{j}-\Omega_{0}^{2} M_{i j}, \\
H_{i j}^{s}= & -h_{0} \Omega_{0} L_{0} \delta_{i}^{j},
\end{aligned}
$$

where $i, j=1, \ldots, s$. For this example, if $s=3$, then all of the submatrices will be of size $3 \times 3$. For definiteness, the original or unperturbed system parameters are $m_{0}=1 \mathrm{~kg} / \mathrm{m}$, $M_{0}=10 \mathrm{~kg}, L_{0}=1 \mathrm{~m}, \mathrm{EI}_{0 x}=8.10 \times 10^{-2} \mathrm{~N} \cdot \mathrm{m}^{2}, \mathrm{EI}_{0 y}=1.82 \times$ $10^{-1} \mathrm{~N} \cdot \mathrm{m}^{2}, \quad K_{01}=K_{02}=5 \times 10^{-2} \mathrm{~N} / \mathrm{m}, \quad c_{0}=h_{0}=2.5 \times 10^{-1}$ $\mathrm{N} \cdot \mathrm{s} / \mathrm{m}, \Omega_{0}=1.46 \times 10^{1} \mathrm{rad} / \mathrm{s}$. The eigensolutions of the unperturbed system are obtained using the state vector approach, where the system matrices are given by

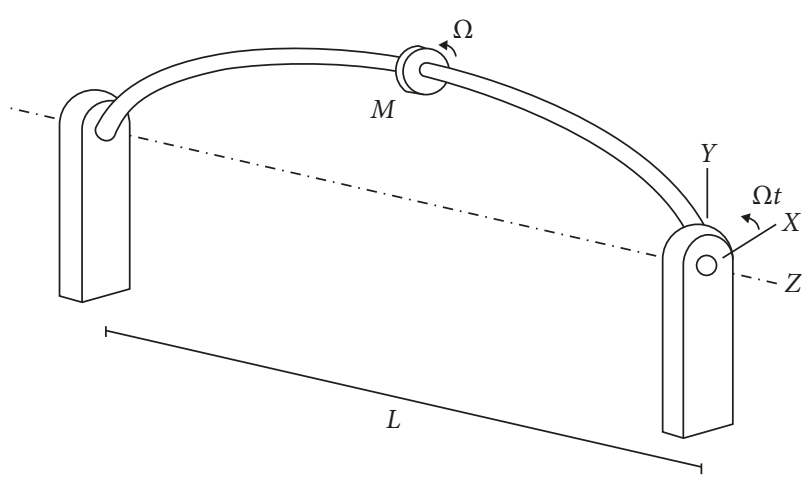

FIGURE 11: A whirling beam.

$$
\begin{aligned}
& {\left[A_{0}\right]=\left[\begin{array}{cc}
{[0]} & {\left[M_{0}\right]} \\
{\left[M_{0}\right]} & {\left[C_{0}\right]+\left[G_{0}\right]}
\end{array}\right],} \\
& {\left[B_{0}\right]=\left[\begin{array}{cc}
{\left[M_{0}\right]} & {[0]} \\
{[0]} & -\left[K_{0}\right]-\left[H_{0}\right]
\end{array}\right] .}
\end{aligned}
$$

Assume now the parameters affecting the damping matrices $\left[C_{0}\right]$ and $\left[G_{0}\right]$ are perturbed so that the modified parameters are given by $M=M_{0}(1+\sigma \delta M)$, $m=m_{0}(1+\sigma \delta m), \quad c=c_{0}(1+\sigma \delta c)$ and $h=h_{0}(1+\sigma \delta h)$, where $\delta M=-1.1079 \times 10^{0}, \quad \delta m=2.5260 \times 10^{-1}$, $\delta c=2.4600 \times 10^{-2}$, and $\delta h=-1.1270 \times 10^{0}$. For the given set of system parameters, $\lambda_{1}$ and $\lambda_{2}$ are strictly real while the remaining eigenvalues are complex. Additionally, the real components of the first and fourth eigenvalues are positive, indicating that the system is unstable. Figures 12 and 13 illustrate the real and imaginary parts of the eigenvalues as a function of $\sigma$, obtained using the methods developed in this paper. The diagonal elements of the second-order triple product again track the exact eigenvalues most closely for all values of $\sigma$. Tables 7 and 8 tabulate the numerical results for $\sigma=0.2$. For the given set of random variations, the parameters $M, m, c$, and $h$ deviate from their nominal values by approximately $-22.1 \%, 5.05 \%, 0.49 \%$, and $-22.5 \%$, respectively. Note that the percentage errors for the diagonal elements of Equation (40) with the second-order perturbed modal matrices are all under $0.004 \%$, despite the large deviations in some of the selected system parameters.

Consider now a larger disorder strength of $\sigma=0.5$. Figures 14 and 15 show the real and imaginary parts of the approximate eigenvalues found using the incremental perturbation method and the triple product method as a function of $N$. Note that as $N$ increases, all the approximate eigenvalues converge to the exact results. Tables 9 and 10 tabulate the real and imaginary parts of the eigenvalues for $N=7$ so that the perturbations at each iteration are less than $10 \%$ of the original system matrices. The percentage errors for all five eigenvalues obtained using the second-order triple product method are below $0.005 \%$. These results are substantially more accurate than those obtained using the second-order 


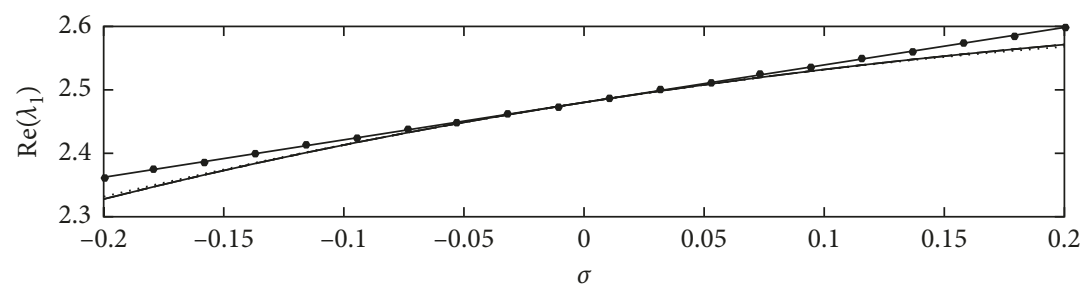

(a)

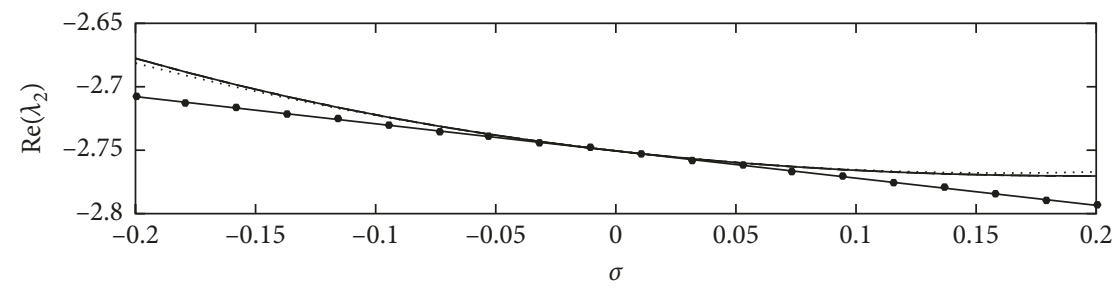

(b)

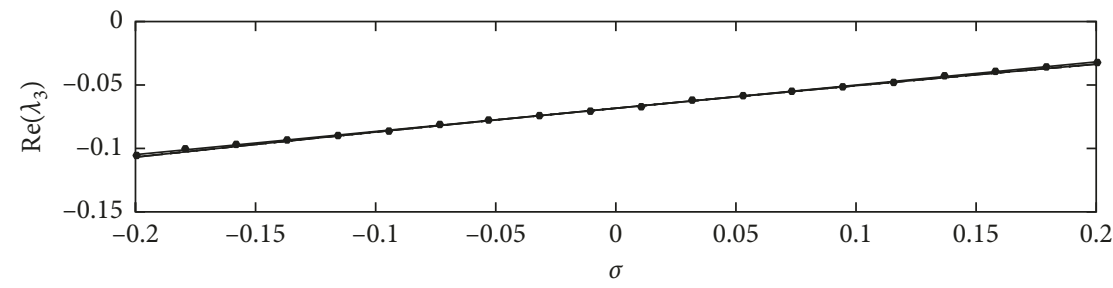

(c)

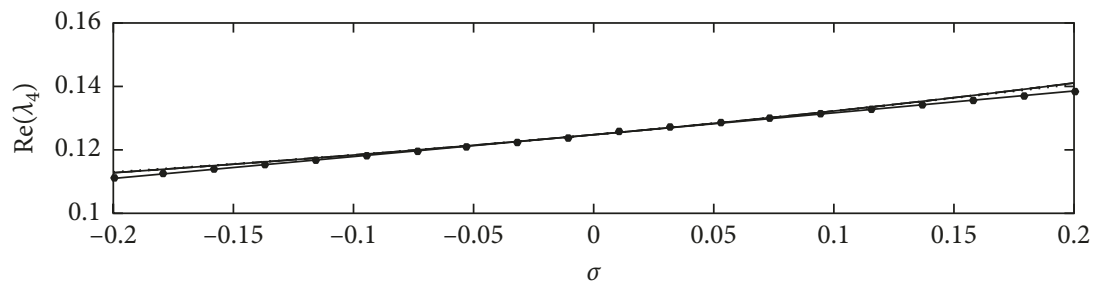

(d)

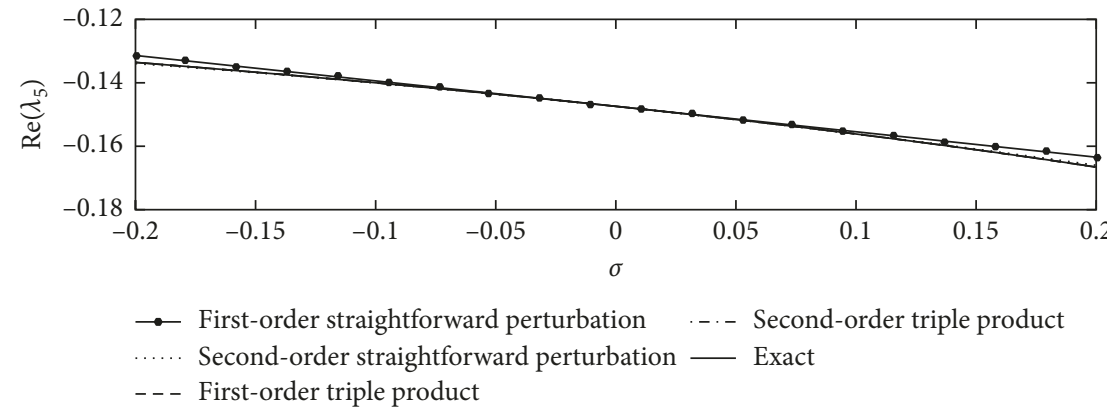

(e)

FIGURE 12: The real parts of the exact and approximate eigenvalues of the whirling beam as a function of $\sigma$.

incremental perturbation method, at the cost of one additional computation given by Equation (40). Figure 16 shows the error parameter $\delta$ for the first-order and second-order perturbed modal matrices (with $p=6$ ) as a function of $N$. Note that as expected, $\delta$ decreases with increasing $N$, and that a second-order analysis is more accurate.
3.4. Nonuniform Rod. The perturbation method can also be applied to analyze the eigencharacteristics of a nonuniform rod. Specifically, the uniform rod will be considered as the unperturbed system, and any deviations from it will be treated as perturbations. Figure 17 shows a nonuniform rod of length $L$, modulus $E$, density $\rho$, and cross-sectional area, $A(x)$, that varies as a function of $x$ from the fixed wall as follows: 


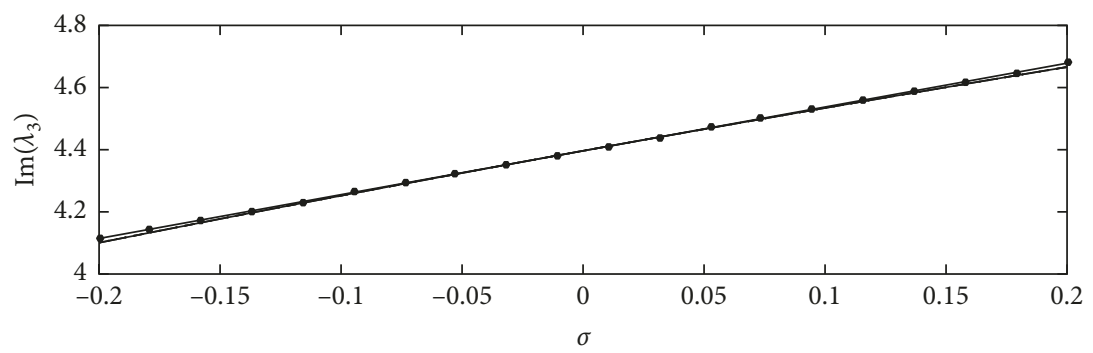

(a)

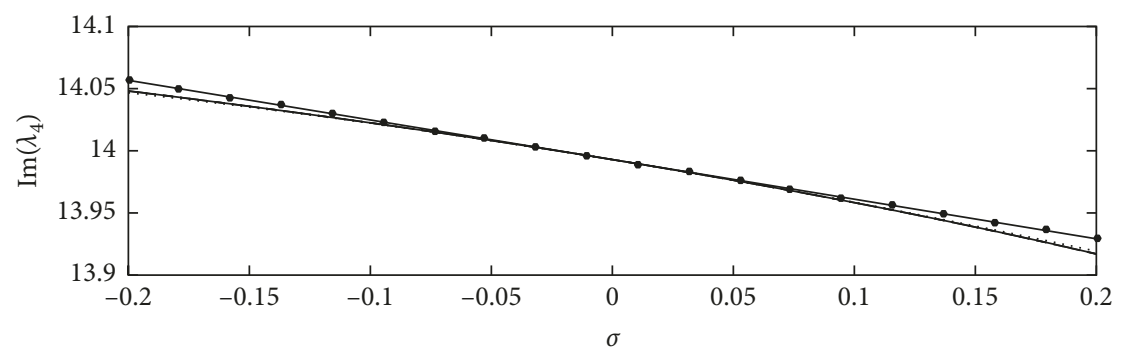

(b)

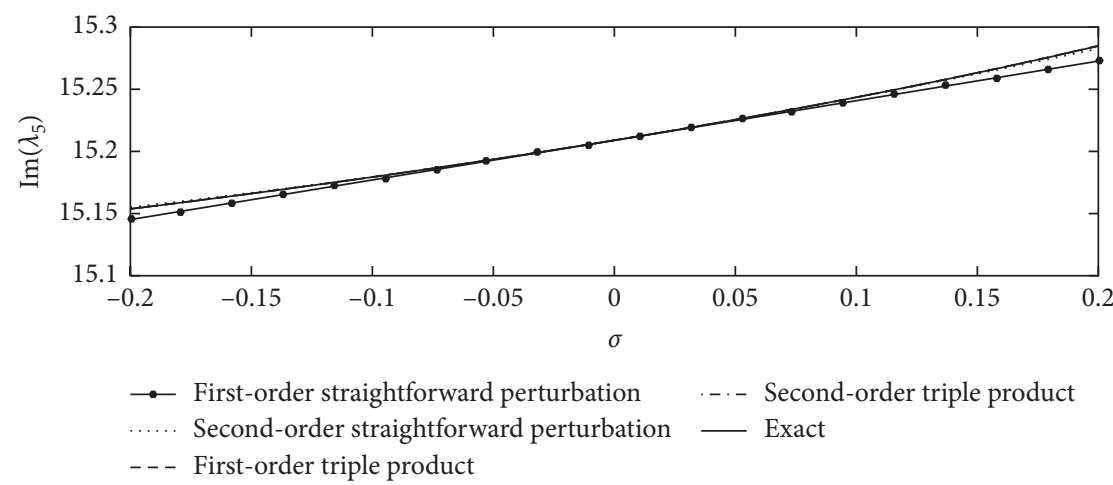

(c)

FIGURE 13: The imaginary parts of the exact and approximate eigenvalues of the whirling beam as a function of $\sigma$.

TABLE 7: The real parts of the exact and approximate natural frequencies for the perturbed whirling beam of Figure 11 for $\sigma=0.2$.

\begin{tabular}{|c|c|c|c|c|c|}
\hline & Exact & $\begin{array}{c}\text { First-order } \\
\text { straightforward } \\
\text { perturbation }\end{array}$ & $\begin{array}{l}\text { Second-order } \\
\text { straightforward } \\
\text { perturbation }\end{array}$ & First-order triple product & $\begin{array}{l}\text { Second-order triple } \\
\text { product }\end{array}$ \\
\hline $\operatorname{Re}\left(\lambda_{1}\right)$ & $2.5713 \times 10^{0}$ & $2.5983 \times 10^{0}\left(1.04 \times 10^{0}\right)$ & $2.5679 \times 10^{0}\left(1.32 \times 10^{-1}\right)$ & $2.5714 \times 10^{0}\left(3.18 \times 10^{-3}\right)$ & $2.5713 \times 10^{0}\left(1.38 \times 10^{-3}\right)$ \\
\hline $\operatorname{Re}\left(\lambda_{2}\right)$ & $-2.7703 \times 10^{0}$ & $\begin{array}{c}-2.7934 \times 10^{0} \\
\left(8.33 \times 10^{-1}\right)\end{array}$ & $\begin{array}{r}-2.7670 \times 10^{0} \\
\left(1.17 \times 10^{-1}\right)\end{array}$ & $\begin{array}{r}-2.7703 \times 10^{0} \\
\left(1.62 \times 10^{-3}\right)\end{array}$ & $\begin{array}{r}-2.7703 \times 10^{0} \\
\left(1.48 \times 10^{-3}\right)\end{array}$ \\
\hline $\operatorname{Re}\left(\lambda_{3}\right)$ & $-3.3574 \times 10^{-2}$ & $\begin{array}{c}-3.1784 \times 10^{-2} \\
\left(5.33 \times 10^{0}\right)\end{array}$ & $\begin{array}{c}-3.3666 \times 10^{-2} \\
\left(2.72 \times 10^{-1}\right)\end{array}$ & $\begin{array}{c}-3.3579 \times 10^{-2} \\
\left(1.41 \times 10^{-2}\right)\end{array}$ & $\begin{array}{c}-3.3574 \times 10^{-2} \\
\left(6.39 \times 10^{-4}\right)\end{array}$ \\
\hline $\operatorname{Re}\left(\lambda_{4}\right)$ & $1.4107 \times 10^{-1}$ & $\begin{array}{c}1.3856 \times 10^{-1} \\
\left(1.78 \times 10^{0}\right)\end{array}$ & $\begin{array}{l}1.4064 \times 10^{-1} \\
\left(3.06 \times 10^{-1}\right)\end{array}$ & $\begin{array}{l}1.4110 \times 10^{-1} \\
\left(2.34 \times 10^{-2}\right)\end{array}$ & $\begin{array}{l}1.4107 \times 10^{-1} \\
\left(2.92 \times 10^{-3}\right)\end{array}$ \\
\hline $\operatorname{Re}\left(\lambda_{5}\right)$ & $-1.6655 \times 10^{-1}$ & $\begin{array}{c}-1.6344 \times 10^{-1} \\
\left(1.86 \times 10^{0}\right)\end{array}$ & $\begin{array}{c}-1.6599 \times 10^{-1} \\
\left(3.34 \times 10^{-1}\right)\end{array}$ & $\begin{array}{c}-1.6660 \times 10^{-1} \\
\left(2.97 \times 10^{-2}\right)\end{array}$ & $\begin{array}{c}-1.6656 \times 10^{-1} \\
\left(3.76 \times 10^{-3}\right)\end{array}$ \\
\hline
\end{tabular}

where $A_{0}, a$, and $b$ are constants. Using the assumed modes method [28], the longitudinal displacement $w(x, t)$ of the rod can be expressed in the form of a finite series as follows: where $s$ is the number of modes used in the expansion, $u_{i}(x)$ are the eigenfunctions of the uniform rod, and $\eta_{i}(t)$ are the 
TABLE 8: The imaginary parts of the exact and approximate natural frequencies for the perturbed whirling beam of Figure 11 for $\sigma=0.2$.

\begin{tabular}{|c|c|c|c|c|c|}
\hline & Exact & $\begin{array}{l}\text { First-order straightforward } \\
\text { perturbation }\end{array}$ & $\begin{array}{l}\text { shtforward } \\
\text { on }\end{array}$ & First-order triple product & $\begin{array}{l}\mathrm{r} \text { triple } \\
\mathrm{t}\end{array}$ \\
\hline & & 200 (a) 19011 & $\left.3 \times 10^{-2}\right)$ & & 4.6661 \\
\hline & & $3929 \times 10^{1}\left(8.71 \times 10^{-2}\right)$ & )$^{1}(1.5$ & 3 ) & 1.3917 \\
\hline $\mathrm{Im}$ & 5284 & $1.5272 \times 10^{1}(7.93 \times$ & $1.5282 \times 10^{1}\left(1.39 \times 10^{-2}\right)$ & $1.5284 \times 10^{1}\left(1.14 \times 10^{-3}\right)$ & $1.5284 \times 10^{1}\left(1.45 \times 10^{-4}\right)$ \\
\hline
\end{tabular}

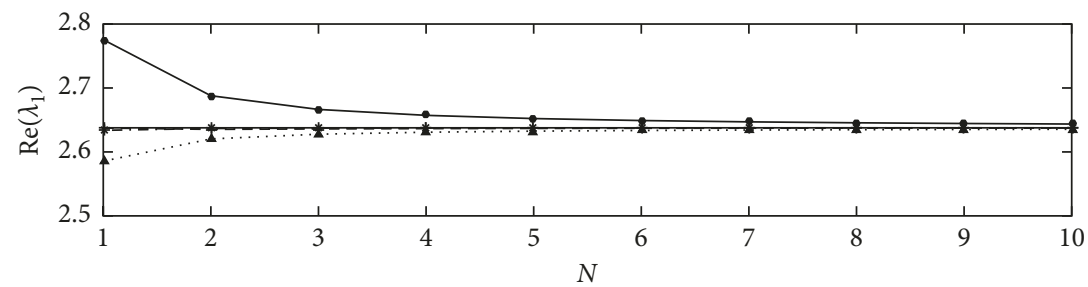

(a)

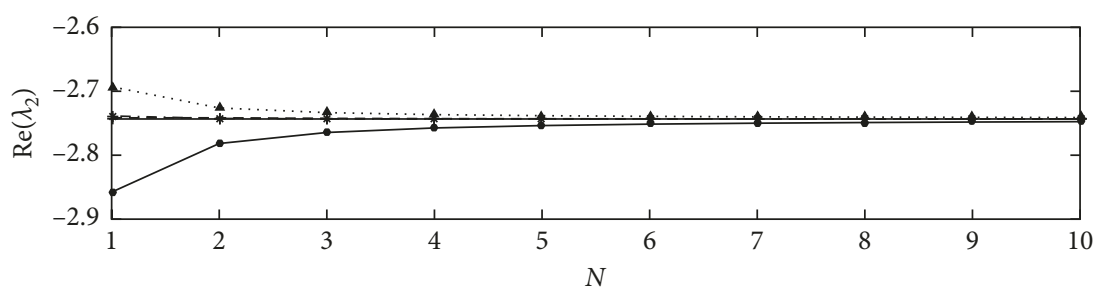

(b)

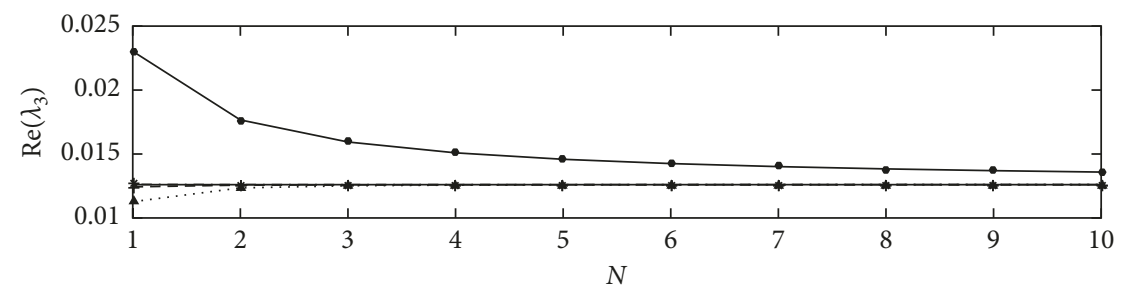

(c)

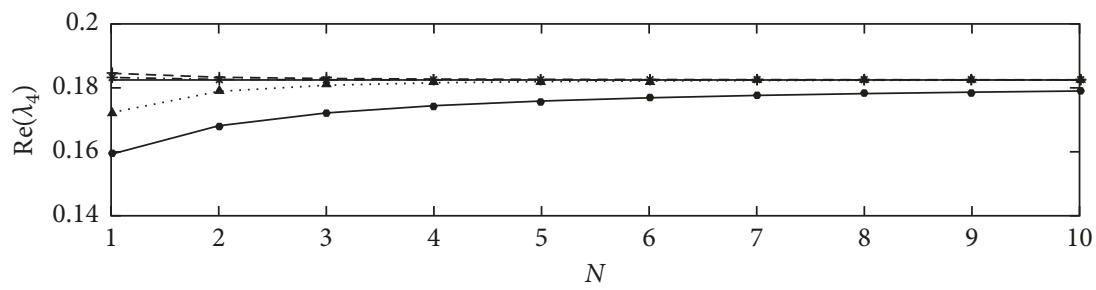

(d)

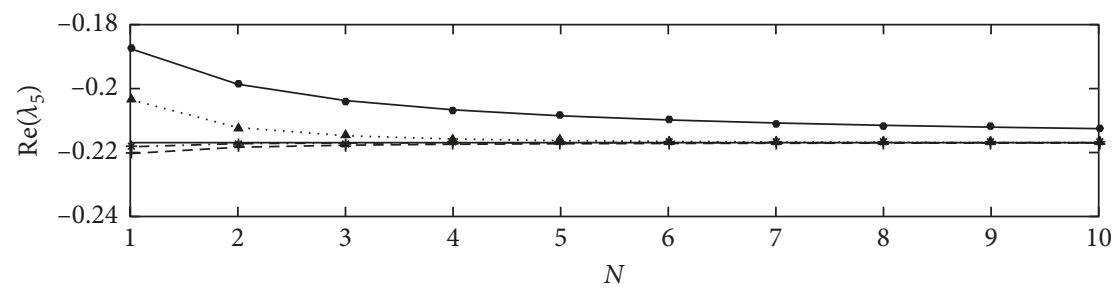

$\rightarrow$ First-order incremental perturbation $\quad . \rightarrow-$ Second-order triple product

... Second-order incremental perturbation

Exact

- + - First-order triple product

(e)

FIGURE 14: The real parts of the approximate eigenvalues of the whirling beam as a function of $N$ for $\sigma=0.5$. 


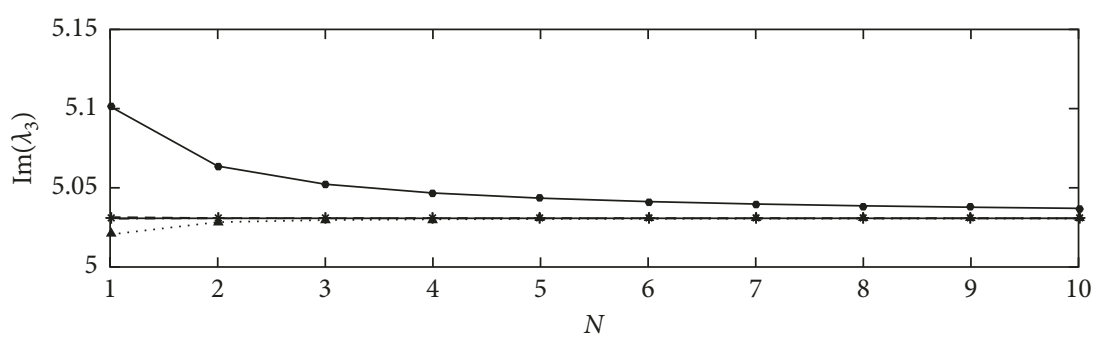

(a)

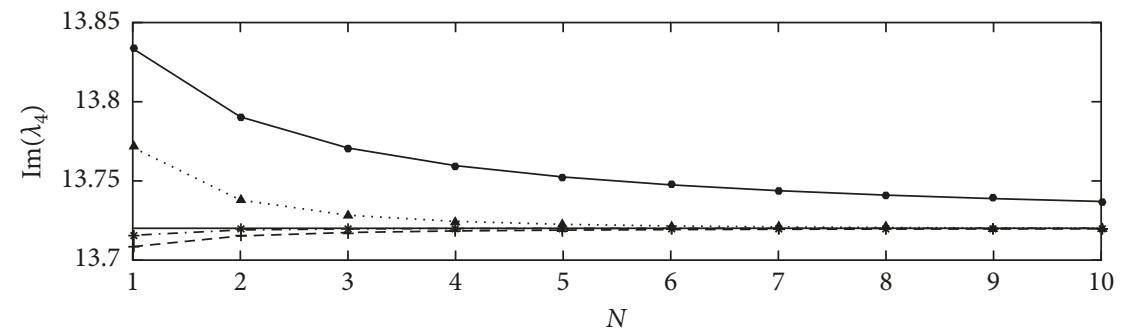

(b)

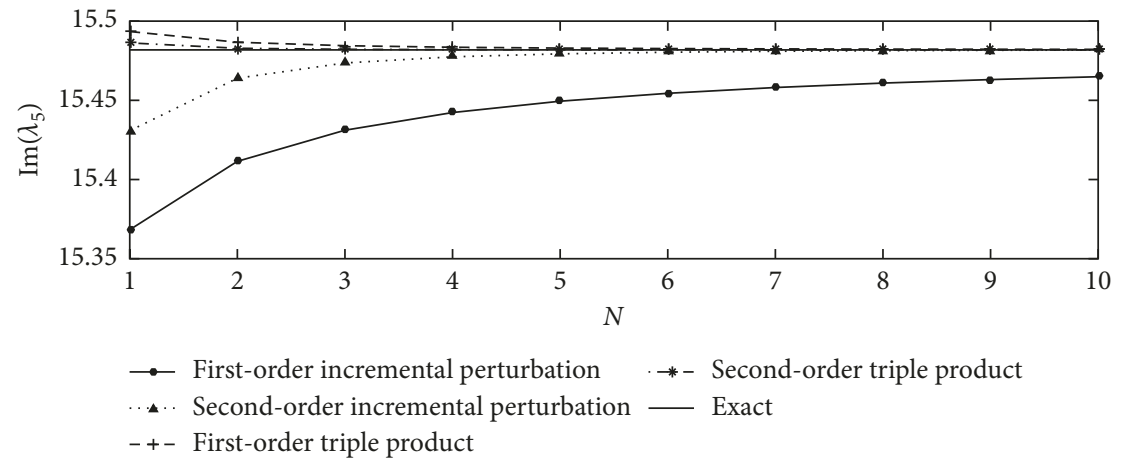

(c)

FIGURE 15: The imaginary parts of the approximate eigenvalues of the whirling beam as a function of $N$ for $\sigma=0.5$.

TABLE 9: The real parts of the exact and approximate natural frequencies for the perturbed whirling beam at $N=7$ for $\sigma=0.5$.

\begin{tabular}{|c|c|c|c|c|c|}
\hline & Exact & $\begin{array}{c}\text { First-order incremental } \\
\text { perturbation }\end{array}$ & $\begin{array}{c}\text { Second-order incremental } \\
\text { perturbation }\end{array}$ & First-order triple product & $\begin{array}{l}\text { Second-order triple } \\
\text { product }\end{array}$ \\
\hline $\operatorname{Re}\left(\lambda_{1}\right)$ & $2.6377 \times 10^{0}$ & $\begin{array}{l}2.6470 \times 10^{0} \\
\left(3.53 \times 10^{-1}\right)\end{array}$ & $\begin{array}{l}2.6342 \times 10^{0} \\
\left(1.30 \times 10^{-1}\right)\end{array}$ & $\begin{array}{l}2.6371 \times 10^{0} \\
\left(2.07 \times 10^{-2}\right)\end{array}$ & $2.6376 \times 10^{0} \quad\left(2.05 \times 10^{-3}\right)$ \\
\hline $\operatorname{Re}\left(\lambda_{2}\right)$ & $-2.7433 \times 10^{0}$ & $\begin{array}{r}-2.7499 \times 10^{0} \\
\left(2.40 \times 10^{-1}\right)\end{array}$ & $\begin{array}{r}-2.7399 \times 10^{0} \\
\left(1.23 \times 10^{-1}\right)\end{array}$ & $\begin{array}{r}-2.7432 \times 10^{0} \\
\left(4.96 \times 10^{-3}\right)\end{array}$ & $-2.7433 \times 10^{0}\left(5.26 \times 10^{-4}\right)$ \\
\hline $\operatorname{Re}\left(\lambda_{3}\right)$ & $1.2603 \times 10^{-2}$ & $\begin{array}{c}1.4014 \times 10^{-2} \\
\left(1.11 \times 10^{1}\right)\end{array}$ & $\begin{array}{l}1.2605 \times 10^{-2} \\
\left(1.42 \times 10^{-2}\right)\end{array}$ & $\begin{array}{l}1.2600 \times 10^{-2} \\
\left(2.04 \times 10^{-2}\right)\end{array}$ & $1.2603 \times 10^{-2}\left(1.45 \times 10^{-4}\right)$ \\
\hline $\operatorname{Re}\left(\lambda_{4}\right)$ & $1.8246 \times 10^{-1}$ & $\begin{array}{c}1.7763 \times 10^{-1} \\
\left(2.64 \times 10^{0}\right)\end{array}$ & $\begin{array}{c}1.8228 \times 10^{-1} \\
\left(1.02 \times 10^{-1}\right)\end{array}$ & $\begin{array}{c}1.8257 \times 10^{-1} \\
\left(5.89 \times 10^{-2}\right)\end{array}$ & $1.8247 \times 10^{-1}\left(2.45 \times 10^{-3}\right)$ \\
\hline $\operatorname{Re}\left(\lambda_{5}\right)$ & $-2.1690 \times 10^{-1}$ & $\begin{array}{c}-2.1075 \times 10^{-1} \\
\left(2.83 \times 10^{0}\right)\end{array}$ & $\begin{array}{c}-2.1669 \times 10^{-1} \\
\left(9.61 \times 10^{-2}\right)\end{array}$ & $\begin{array}{c}-2.1710 \times 10^{-1} \\
\left(8.96 \times 10^{-2}\right)\end{array}$ & $-2.1691 \times 10^{-1}\left(4.37 \times 10^{-3}\right)$ \\
\hline
\end{tabular}

TABLE 10: The imaginary parts of the exact and approximate natural frequencies for the perturbed whirling beam at $N=7$ for $\sigma=0.5$.

\begin{tabular}{|c|c|c|c|c|c|}
\hline & Exact & $\begin{array}{l}\text { First-order incremental } \\
\text { perturbation }\end{array}$ & $\begin{array}{l}\text { Second-order incremental } \\
\text { perturbation }\end{array}$ & First-order triple product & erond order \\
\hline & & $0397 \times 10^{0}$ & $5.0304 \times 10^{0}$ & & 5.0 \\
\hline & 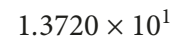 & $1.3743 \times 10^{1}(1.73 \times$ & $1.3721 \times 10^{1}(6.34 \times$ & $1.3719 \times 10^{1}(4.66)$ & $1.3720 \times 10^{1}(2.16$ \\
\hline$\left(\lambda_{5}\right)$ & $.5481 \times 10^{1}$ & $1.5458 \times 10^{1}\left(1.53 \times 10^{-1}\right)$ & $1.5480 \times 10^{1}\left(5.61 \times 10^{-3}\right)$ & $1.5482 \times 10^{1}\left(4.13 \times 10^{-3}\right)$ & $1.5481 \times 10^{1}\left(1.92 \times 10^{-4}\right)$ \\
\hline
\end{tabular}




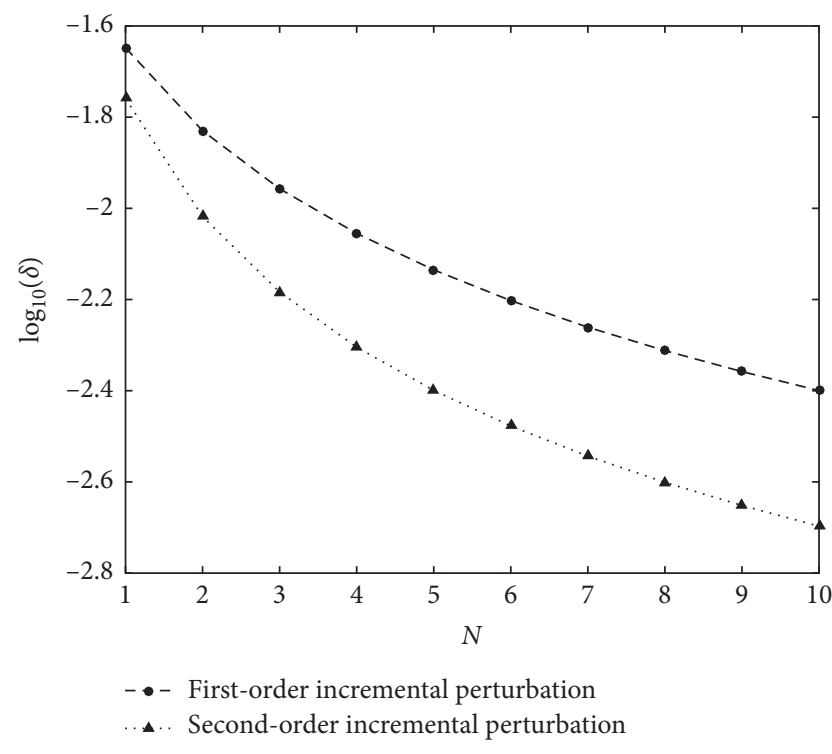

FIgURE 16: The error parameter, $\delta$, for the perturbed modal matrices of the whirling beam as a function of $N$ for $\sigma=0.5$.

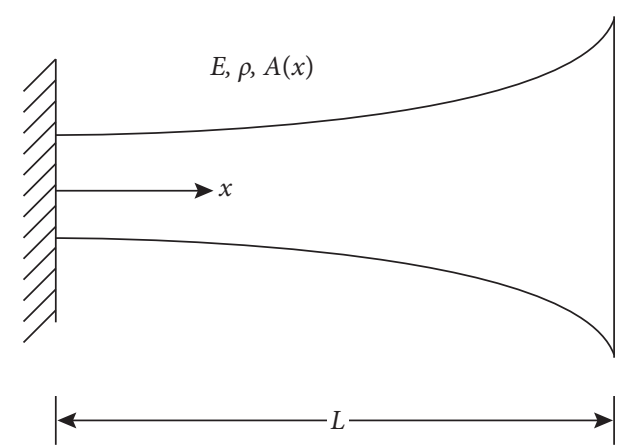

FIgURE 17: A nonuniform rod.

unknown generalized coordinates. Formulating the total kinetic and potential energies of the system, one obtains

$$
\begin{aligned}
& T=\frac{1}{2} \sum_{i=1}^{s} \sum_{j=1}^{s} M_{i j} \dot{\eta}_{i}(t) \dot{\eta}_{j}(t), \\
& U=\frac{1}{2} \sum_{i=1}^{s} \sum_{j=1}^{s} K_{i j} \eta_{i}(t) \eta_{j}(t),
\end{aligned}
$$

where the generalized masses and stiffnesses of the nonuniform rod are given by

$$
\begin{aligned}
M_{i j} & =\int_{0}^{L} \rho A(x) u_{i}(x) u_{j}(x) d x, \\
K_{i j} & =\int_{0}^{L} E A(x) \frac{d u_{i}(x)}{d x} \frac{d u_{j}(x)}{d x} d x .
\end{aligned}
$$

Applying Lagrange's equations yields the following discretized equations of motion:

$$
[M] \ddot{\boldsymbol{\eta}}+[K] \boldsymbol{\eta}=0,
$$

where the $(i, j)$ th elements of matrices $[M]$ and $[K]$ are $M_{i j}$ and $K_{i j}$, respectively. Moreover, note that $[M]$ and $[K]$ are both symmetric, and because $s$ modes are used in the assumed modes expansion, they are of size $s \times s$.

For this particular system, the uniform rod whose crosssectional area is $A_{0}$ represents the unperturbed system, and the nonuniform rod is the perturbed system. For a uniform fixed-free rod, its normalized eigenfunctions are

$$
u_{i}(x)=\sqrt{\frac{2}{\rho A_{0} L}} \sin \left(\frac{(2 i-1) \pi x}{2 L}\right),
$$

and its natural frequencies are

$$
\omega_{0 i}=\frac{(2 i-1) \pi}{2} \sqrt{\frac{E}{\rho L^{2}}} .
$$

Because the unperturbed system consists of the uniform rod, the unperturbed matrices are

$$
\begin{aligned}
{\left[M_{0}\right] } & =[I], \\
{\left[K_{0}\right] } & =\left[\Lambda_{0}\right],
\end{aligned}
$$

where $[I]$ denotes the identity matrix, and $\left[\Lambda_{0}\right]$ is a diagonal matrix whose elements are $\omega_{0 i}^{2}$. For this undamped system, $\lambda=\omega^{2}$. Finally, Equations (33)-(35) still remain valid. In this case, of course, $[A]=[M]$ and $[B]=[K]$. Note that unlike the previous examples, $[A]$ and $[B]$ are both symmetric.

For definiteness, consider the case where the crosssectional area varies as a function of $x$ with $b=2$ in Equation (53). In this case, the exact natural frequencies for the nonuniform rod are given by the solution of the following transcendental equation (see [29] for detailed derivations)

$$
\frac{a}{1+a} \tan \beta_{i}=\beta_{i}
$$

where

$$
\omega_{i}=\beta_{i} \sqrt{\frac{E}{\rho L^{2}}} .
$$

Figure 18 shows the natural frequencies as a function of $a$, where $a$ spans $-0.35 \leq a \leq 0.35$, obtained using $s=10$ in the assumed modes expansion. For $a=0.35$, the rod's cross-sectional area at its free end is approximately $82 \%$ larger than its cross-sectional area at the base. Again, the natural frequencies obtained using the triple product method closely track the exact natural frequencies. The results are tabulated in Table 11. Although a $82 \%$ change in the rod's cross-sectional area is significant, note that the largest error parameter for the first-order triple product method is less than $0.32 \%$, while for the second-order triple product method it is less than $0.06 \%$.

Consider now a larger nonuniformity with $a=0.55$, implying that the cross-sectional area at the free end of the rod is $140 \%$ greater than its cross-sectional area at the fixed end. An incremental perturbation approach is used to obtain the approximate natural frequencies. Figure 19 


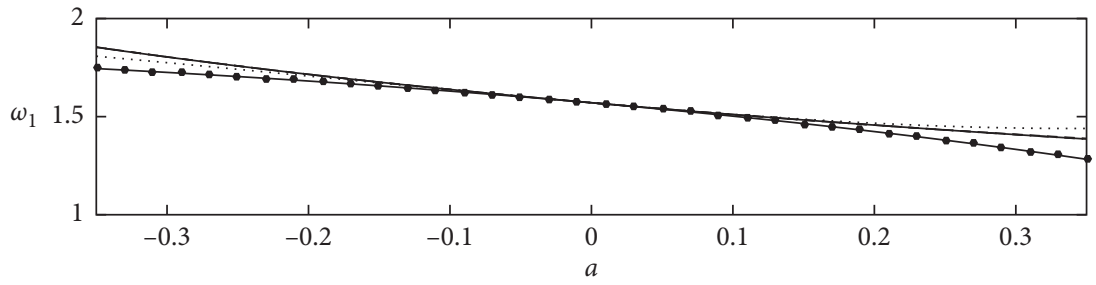

(a)

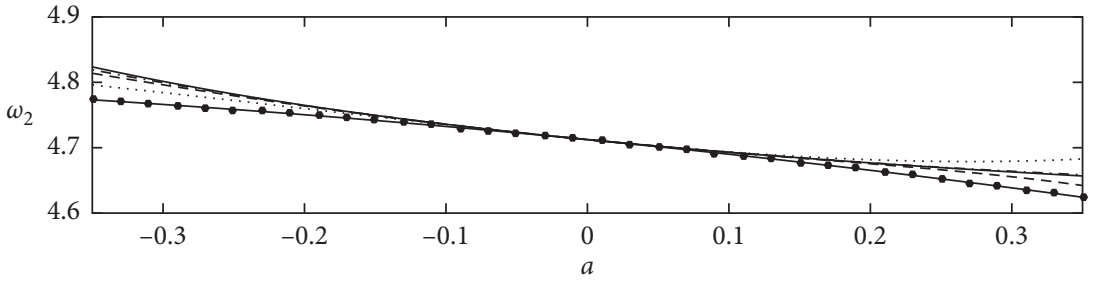

(b)

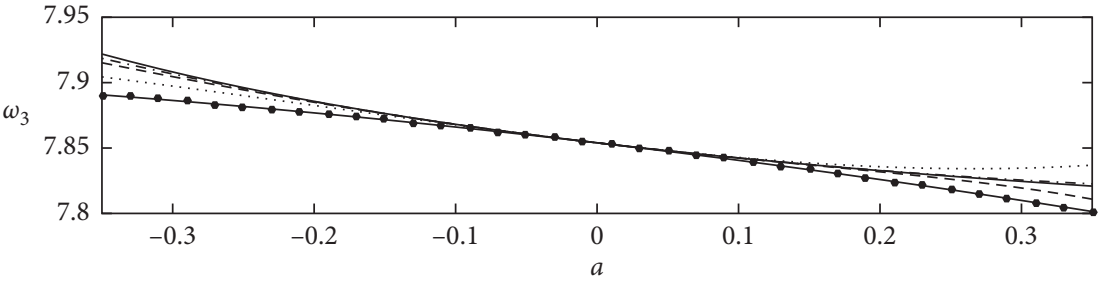

(c)

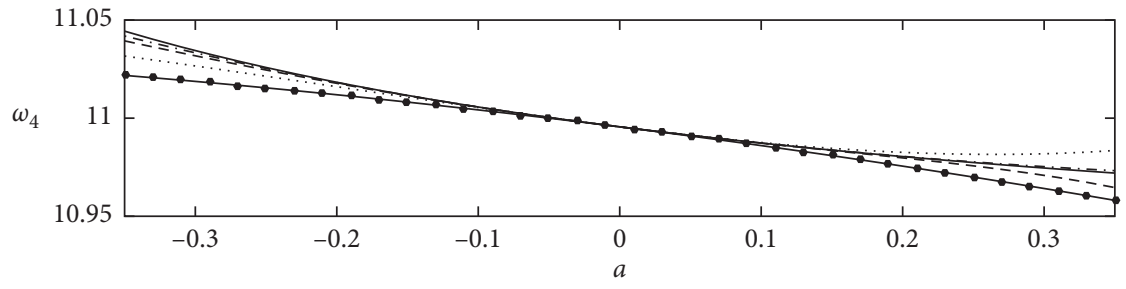

(d)

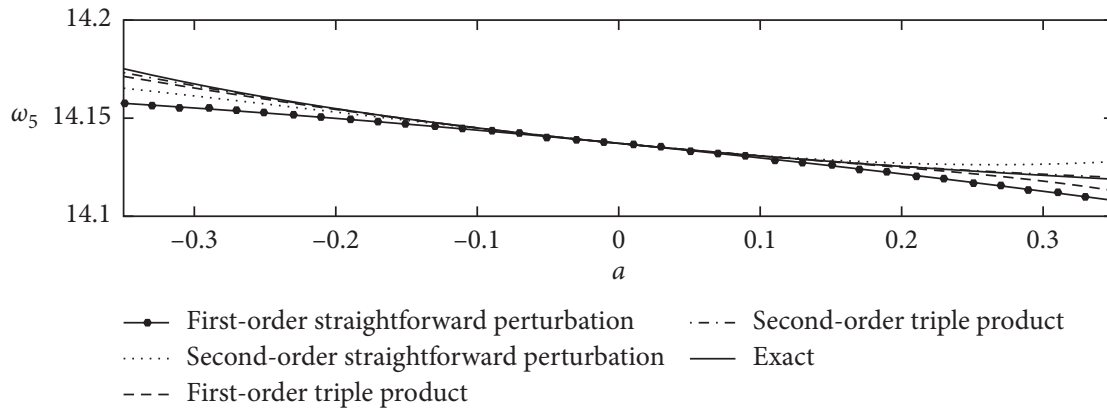

(e)

Figure 18: The first five normalized exact and approximate natural frequencies of the nonuniform rod as a function of $a$. The natural frequencies are nondimensionalized by dividing by $\sqrt{E /\left(\rho L^{2}\right)}$.

shows the approximate natural frequencies obtained using the same five methods as a function of $N$. Although the approximate natural frequencies are inaccurate when $N=$ 1 or $N=2$, they quickly converge to the exact natural frequencies as $N$ continues to increase. Table 12 tabulates the perturbed natural frequencies of Figure 19 for $N=10$ so that the perturbation at each iteration is less than $10 \%$ of the original system matrices. Note that the percentage errors, $\varepsilon$, of the first-order and second-order triple product approximations for all five natural frequencies are less than $0.006 \%$. It should be noted that for this example, because the nonuniform rod has been discretized using the assumed modes method, the perturbed natural frequencies will converge to the natural frequencies of the discretized and not the continuum model of the nonuniform rod. As such, $\omega_{3}$ to $\omega_{5}$ obtained using the first-order triple 
TABLE 11: The exact and approximate natural frequencies for the nonuniform rod of Figure 17 for $a=0.35$. The natural frequencies are nondimensionalized by dividing by $\sqrt{E /\left(\rho L^{2}\right)}$.

\begin{tabular}{cccccccc}
\hline & Exact & $\begin{array}{c}\text { First-order straightforward } \\
\text { perturbation }\end{array}$ & $\begin{array}{c}\text { Second-order straightforward } \\
\text { perturbation }\end{array}$ & First-order triple product & Second-order triple product \\
\hline$\omega_{1}$ & $1.3858 \times 10^{0}$ & $1.2825 \times 10^{0}\left(7.45 \times 10^{0}\right)$ & $1.4396 \times 10^{0}\left(3.88 \times 10^{0}\right)$ & $1.3889 \times 10^{0}\left(2.19 \times 10^{-1}\right)$ & $1.3851 \times 10^{0}\left(5.22 \times 10^{-2}\right)$ \\
$\omega_{2}$ & $4.6568 \times 10^{0}$ & $4.6242 \times 10^{0}\left(6.98 \times 10^{-1}\right)$ & $4.6828 \times 10^{0}\left(5.58 \times 10^{-1}\right)$ & $4.6422 \times 10^{0}\left(3.12 \times 10^{-1}\right)$ & $4.6586 \times 10^{0}$ & $\left(3.84 \times 10^{-2}\right)$ \\
$\omega_{3}$ & $7.8209 \times 10^{0}$ & $7.8014 \times 10^{0}$ & $\left(2.48 \times 10^{-1}\right)$ & $7.8369 \times 10^{0}\left(2.04 \times 10^{-1}\right)$ & $7.8109 \times 10^{0}\left(1.27 \times 10^{-1}\right)$ & $7.8226 \times 10^{0}$ & $\left(2.20 \times 10^{-2}\right)$ \\
$\omega_{4}$ & $1.0972 \times 10^{1}$ & $1.0958 \times 10^{1}$ & $\left(1.26 \times 10^{-1}\right)$ & $1.0983 \times 10^{1}\left(1.04 \times 10^{-1}\right)$ & $1.0964 \times 10^{1}\left(6.73 \times 10^{-2}\right)$ & $1.0973 \times 10^{1}\left(1.11 \times 10^{-2}\right)$ \\
$\omega_{5}$ & $1.4119 \times 10^{1}$ & $1.4108 \times 10^{1}$ & $\left(7.67 \times 10^{-2}\right)$ & $1.4128 \times 10^{1}\left(6.36 \times 10^{-2}\right)$ & $1.4113 \times 10^{1}\left(4.13 \times 10^{-2}\right)$ & $1.4120 \times 10^{1}$ & $\left(6.93 \times 10^{-3}\right)$ \\
\hline
\end{tabular}

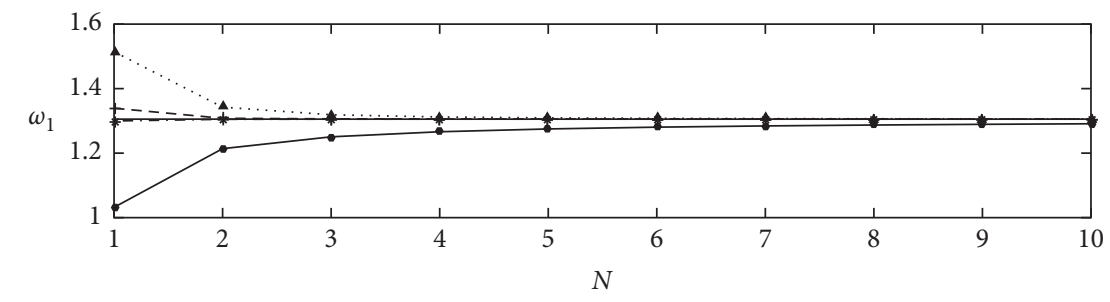

(a)

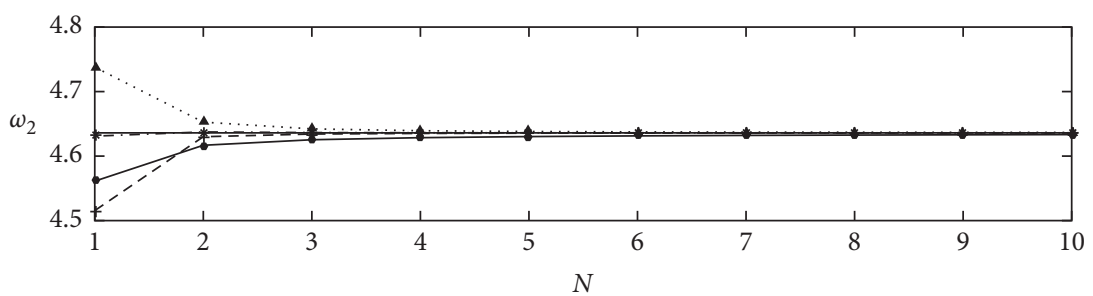

(b)

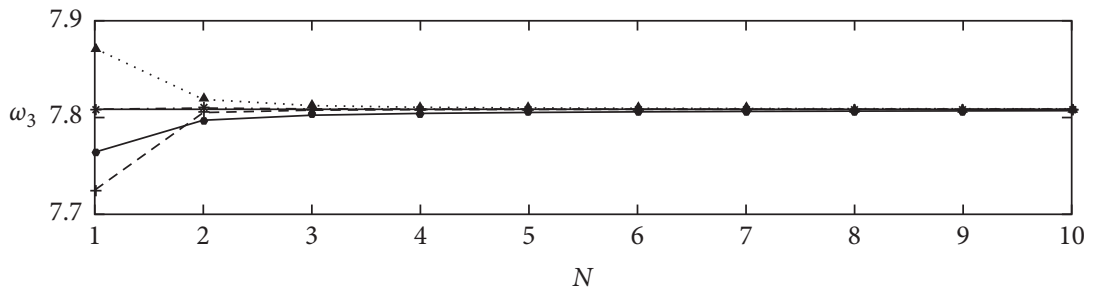

(c)

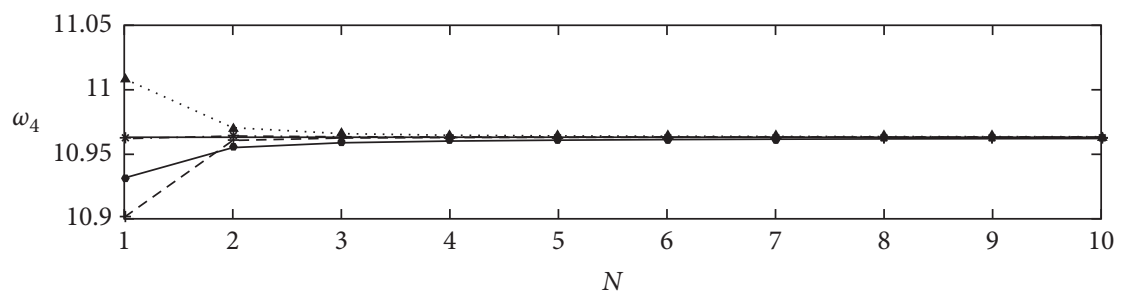

(d)

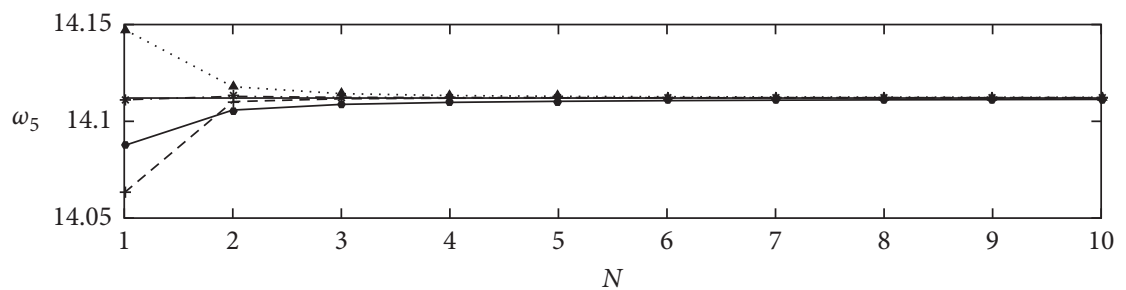

$\rightarrow$ First-order incremental perturbation $-*$ - Second-order triple product
$\ldots$-.. Second-order incremental perturbation - Exact
-+- First-order triple product

(e)

FIGURE 19: The approximate natural frequencies of the nonuniform rod as a function of $N$ for $a=0.55$. The natural frequencies are nondimensionalized by dividing by $\sqrt{E /\left(\rho L^{2}\right)}$. 
TABLE 12: The exact and approximate natural frequencies for the nonuniform rod at $N=10$ for $a=0.55$. The natural frequencies are nondimensionalized by dividing by $\sqrt{E /\left(\rho L^{2}\right)}$.

\begin{tabular}{ccccccc}
\hline & Exact & $\begin{array}{c}\text { First-order incremental } \\
\text { perturbation }\end{array}$ & $\begin{array}{c}\text { Second-order incremental } \\
\text { perturbation }\end{array}$ & First-order triple product & Second-order triple product \\
\hline$\omega_{1}$ & $1.3053 \times 10^{0}$ & $1.2910 \times 10^{0}\left(1.10 \times 10^{0}\right)$ & $1.3060 \times 10^{0}\left(5.31 \times 10^{-2}\right)$ & $1.3054 \times 10^{0}\left(5.56 \times 10^{-3}\right)$ & $1.3053 \times 10^{0}\left(1.10 \times 10^{-3}\right)$ \\
$\omega_{2}$ & $4.6359 \times 10^{0}$ & $4.6333 \times 10^{0}\left(5.79 \times 10^{-2}\right)$ & $4.6366 \times 10^{0}\left(1.42 \times 10^{-2}\right)$ & $4.6359 \times 10^{0}\left(1.40 \times 10^{-3}\right)$ & $4.6360 \times 10^{0}\left(1.18 \times 10^{-3}\right)$ \\
$\omega_{3}$ & $7.8085 \times 10^{0}$ & $7.8070 \times 10^{0}\left(1.95 \times 10^{-2}\right)$ & $7.8090 \times 10^{0}\left(5.59 \times 10^{-3}\right)$ & $7.8085 \times 10^{0}\left(2.47 \times 10^{-4}\right)$ & $7.8086 \times 10^{0}\left(1.07 \times 10^{-3}\right)$ \\
$\omega_{4}$ & $1.0963 \times 10^{1}$ & $1.0962 \times 10^{1}\left(9.09 \times 10^{-3}\right)$ & $1.0963 \times 10^{1}\left(3.65 \times 10^{-3}\right)$ & $1.0963 \times 10^{1}\left(8.79 \times 10^{-4}\right)$ & $1.0963 \times 10^{1}\left(1.30 \times 10^{-3}\right)$ \\
$\omega_{5}$ & $1.4112 \times 10^{1}$ & $1.4111 \times 10^{1}\left(4.98 \times 10^{-3}\right)$ & $1.4112 \times 10^{1}\left(2.75 \times 10^{-3}\right)$ & $1.4112 \times 10^{1}\left(1.07 \times 10^{-3}\right)$ & $1.4112 \times 10^{1}\left(1.33 \times 10^{-3}\right)$ \\
\hline
\end{tabular}

product method with $N=10$ are more accurate than the ones found using the second-order triple product approach. This unexpected result is purely an artifact of the discretization process. If the number of modes, $s$, used in the assumed modes method is sufficiently large, then the secondorder triple product approach will yield natural frequencies that are more accurate than those obtained using a first-order triple product method. Figure 20 shows the error parameters $\delta$ for the corresponding first-order and second-order modal matrices as a function of $N$. Note that for $N=10, \delta<0.001$ for the second-order incremental perturbation method, validating the accuracy of the resulting modal matrices.

Finally, because the computational power of software and hardware these days is high, a few words on the advantages of the proposed methods are warranted. Consider a large, complicated system with a dozen or more parameters. In order to conduct a parametric study on the effects of parameter modifications on the eigenvalues of the system, computationally resolving the eigenvalue problem could be costly and time intensive. The perturbation methods proposed in this paper, on the other hand, can be used efficiently to conduct such an analysis because the proposed methods involve only simple matrix multiplication. In addition to their efficiency, these methods can accurately approximate a perturbed system's eigensolutions. Moreover, the proposed methods are not limited by the magnitude of the perturbations. Using an incremental approach, these methods maintain their efficiency and accuracy even for large modifications. In addition, the perturbation methods yield physical insights into the problem that solving the problem computationally does not. Using the closed-form expressions given in Equations (34) and (35), one can track how changes to an individual parameter affect specific eigenvalues of the system. One can also calculate the rate at which certain eigenvalues change with respect to specific parameters. The perturbation methods can be harnessed to solve challenging inverse problems as well. Specifically, if the system requires specific eigenvalues and eigenvectors, one could determine which parameters must be changed to obtain said specifications. It would be difficult to solve such an inverse problem numerically on a computer because one could not predict how changing certain parameters may affect the system's eigensolutions a priori without guessing and checking.

\section{Conclusion}

In this paper, three methods are proposed based on the eigenvalue perturbation theory to approximate the

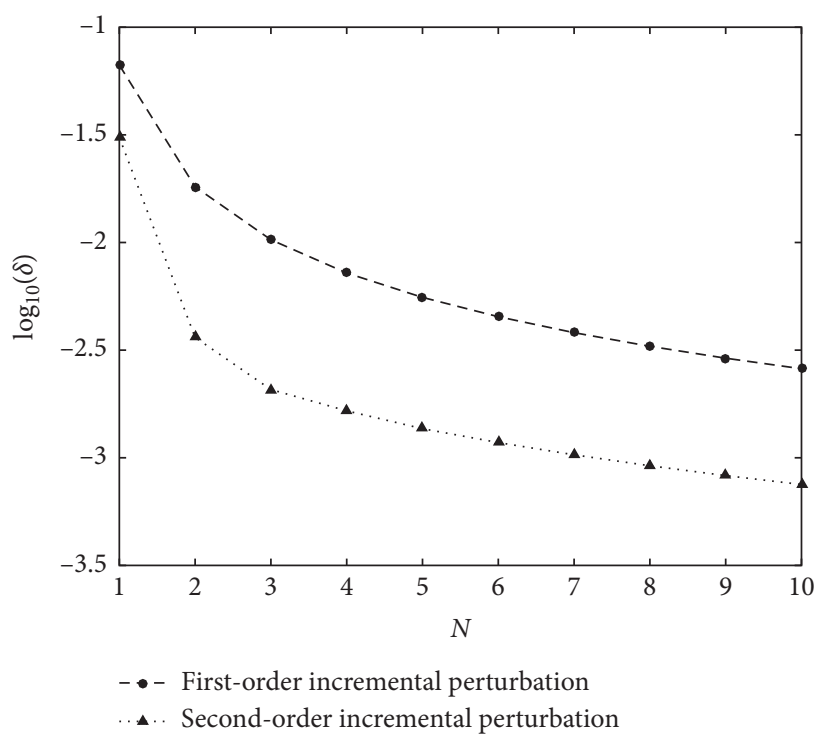

Figure 20: The error parameter, $\delta$, for the perturbed modal matrices of the nonuniform rod as a function of $N$ for $a=0.55$. The natural frequencies are nondimensionalized by dividing by $\sqrt{E /\left(\rho L^{2}\right)}$.

eigencharacteristics of symmetric and asymmetric systems that have been subjected to modifications. These methods only require the eigensolutions of the nominal system, and they involve only simple matrix multiplications. The straightforward perturbation method is easy to implement, simple to code, efficient to apply, and it yields accurate results when the modifications are small. When large design changes are introduced, an incremental perturbation scheme is proposed whereby the large modifications are perturbed incrementally. A triple product method based on the orthogonality conditions of the perturbed eigenvectors offers yet another means to approximate the new eigenvalues of the modified system. It also serves to validate the accuracy of the perturbed eigenvectors. Numerical experiments are performed on various symmetric and asymmetric systems, and they show that the incremental perturbation method and the triple product method can accurately track the exact eigensolutions even when large design changes are introduced. The eigenvalue perturbation theory yields accurate eigencharacteristics and, more importantly, allows the designer to gain physical insights regarding the effects of modifications on the dynamic characteristics of the system. 


\section{Data Availability}

The numerical data used to support the findings of this study are included within the article.

\section{Conflicts of Interest}

The authors declare that there are no conflicts of interest regarding the publication of this paper.

\section{References}

[1] R. L. Fox and M. P. Kapoor, "Rates of change of eigenvalues and eigenvectors," AIAA Journal, vol. 6, no. 12, pp. 24262429, 1968.

[2] K. M. Romstad, J. R. Hutchinson, and K. H. Runge, "Design parameter variation and structural response," International Journal for Numerical Methods in Engineering, vol. 5, no. 3, pp. 337-349, 1973.

[3] R. H. Plaut and K. Huseyin, "Derivatives of eigenvalues and eigenvectors in non-self-adjoint systems," AIAA Journal, vol. 11, no. 2, pp. 250-251, 1973.

[4] C. S. Rudisill, "Derivatives of eigenvalues and eigenvectors for a general matrix," AIAA Journal, vol. 12, no. 5, pp. 721-722, 1974.

[5] L. Meirovitch, "A new method of solution of the eigenvalue problem for gyroscopic systems," AIAA Journal, vol. 12, no. 10 , pp. 1337-1342, 1974.

[6] L. Meirovitch, "A modal analysis for the response of linear gyroscopic systems," Journal of Applied Mechanics, vol. 42, no. 2, pp. 446-450, 1975.

[7] C. S. Rudisill and Y. Y. Chu, "Numerical methods for evaluating the derivatives of eigenvalues and eigenvectors," AIAA Journal, vol. 13, no. 6, pp. 834-836, 1975.

[8] R. B. Nelson, "Simplified calculation of eigenvector derivatives," AIAA Journal, vol. 14, no. 9, pp. 1201-1205, 1976.

[9] J. C. Chen and B. K. Wada, "Matrix perturbation for structural dynamic analysis," AIAA Journal, vol. 15, no. 8, pp. 10951100, 1977.

[10] L. Meirovitch and G. Ryland, "Response of slightly damped gyroscopic systems," Journal of Sound and Vibration, vol. 67, no. 1, pp. 1-19, 1979.

[11] H. V. Belle, "Higher order sensitivities in structural systems," AIAA Journal, vol. 20, no. 2, pp. 286-288, 1982.

[12] L. Meirovitch and G. Ryland, "A perturbation technique for gyroscopic systems with small internal and external damping," Journal of Sound and Vibration, vol. 100, no. 3, pp. 393-408, 1985.

[13] J. F. Baldwin and S. G. Hutton, "Natural modes of modified structures," AIAA Journal, vol. 23, no. 11, pp. 1737-1743, 1985.

[14] K. R. Chung and C. W. Lee, "Dynamic reanalysis of weakly non-proportionally damped systems," Journal of Sound and Vibration, vol. 111, no. 1, pp. 37-50, 1986.

[15] W. B. Bickford, "An improved computational technique for perturbations of the generalized symmetric linear algebraic eigenvalue problem," International Journal for Numerical Methods in Engineering, vol. 24, no. 3, pp. 529-541, 1987.

[16] D. L. Cronin, "Eigenvalue and eigenvector determination for nonclassically damped dynamic systems," Computers and Structures, vol. 36, no. 1, pp. 133-138, 1990.

[17] B. P. Wang, "Improved approximate methods for computing eigenvector derivatives in structural dynamics," AIAA Journal, vol. 29, no. 6, pp. 1018-1020, 1991.
[18] M. S. Eldred, P. B. Lerner, and W. J. Anderson, "Improvement of normalization methods for eigenvector derivatives," AIAA Journal, vol. 30, no. 6, pp. 1609-1616, 1992.

[19] M. S. Eldred, P. B. Lerner, and W. J. Anderson, "Higher order eigenpair perturbations," AIAA Journal, vol. 30, no. 7, pp. 1870-1876, 1992.

[20] M. K. Kwak, "Perturbation method for the eigenvalue problem of lightly damped systems," Journal of Sound and Vibration, vol. 160, no. 2, pp. 351-357, 1993.

[21] S. H. Chen, Z. S. Liu, C. S. Shao, and Y. Q. Zhao, "Perturbation analysis of vibration modes with close frequencies," Communications in Numerical Methods in Engineering, vol. 9, no. 5, pp. 427-438, 1993.

[22] W. Wang and J. Kirkhope, "New eigensolutions and modal analysis for gyroscopic/rotor systems, part 2: perturbation analysis for damped systems," Journal of Sound and Vibration, vol. 175, no. 2, pp. 171-183, 1994.

[23] J. Tang and W. L. Wang, "Perturbation method for determining eigensolutions of weakly damped systems," Journal of Sound and Vibration, vol. 187, no. 4, pp. 671-681, 1995.

[24] J. K. Liu and H. C. Chan, "A universal matrix perturbation technique for structural dynamic modification using singular value decomposition," Journal of Sound and Vibration, vol. 228, no. 2, pp. 265-274, 1999.

[25] N. Trisovic, "Eigenvalue sensitivity analysis in structural dynamics," FME Transactions, vol. 35, no. 3, pp. 149-156, 2007.

[26] P. D. Cha and K. A. Solberg, "Applying eigenvalue perturbation theory to solve problems in structural dynamics," International Journal of Mechanical Engineering Education, vol. 36, no. 2, pp. 160-175, 2008.

[27] E. M. ElBeheiry, "On eigenproblem solution of damped vibrations associated with gyroscopic moments," Journal of Sound and Vibration, vol. 320, no. 3, pp. 691-704, 2009.

[28] L. Meirovitch, Fundamentals of Vibrations, The McGraw-Hill Company, New York, NY, USA, 2001.

[29] S. Abrate, "Vibration of non-uniform rods and beams," Journal of Sound and Vibration, vol. 185, no. 4, pp. 703-716, 1995. 


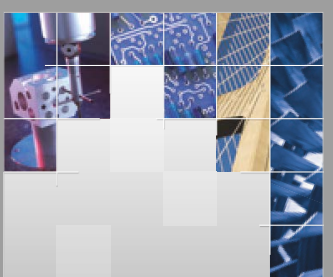

\section{Enfincering}
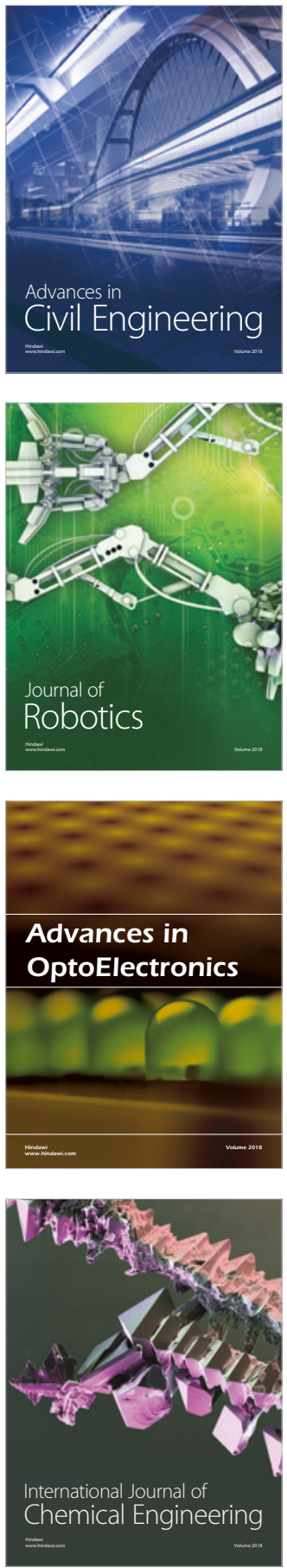

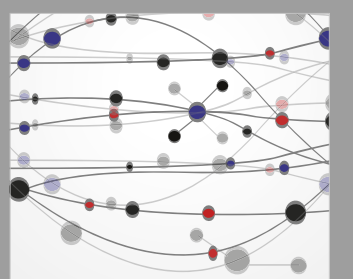

\section{Rotating \\ Machinery}

The Scientific World Journal

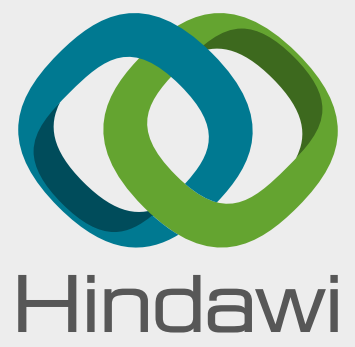

Submit your manuscripts at

www.hindawi.com
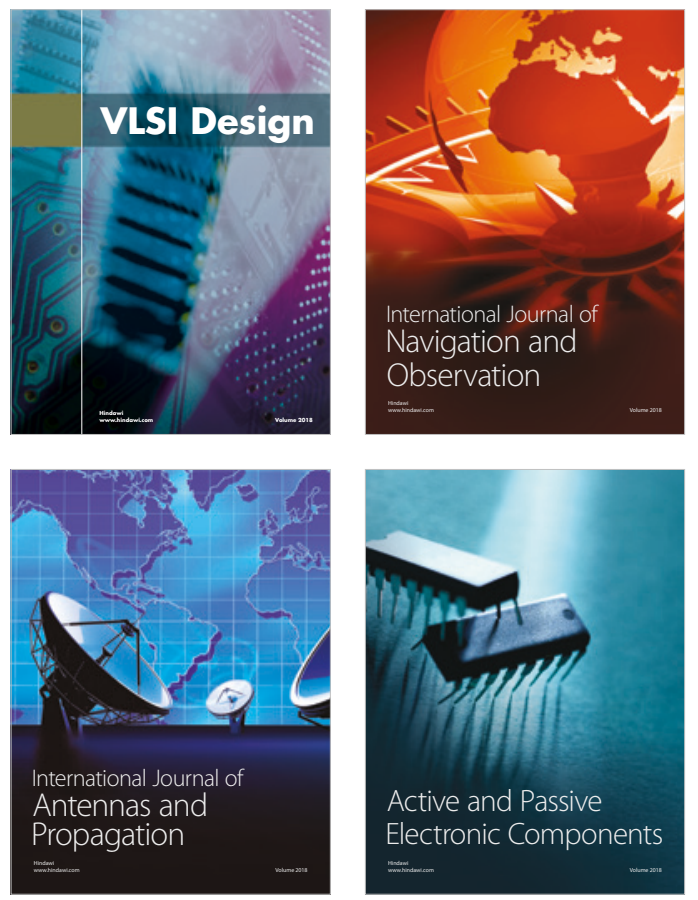
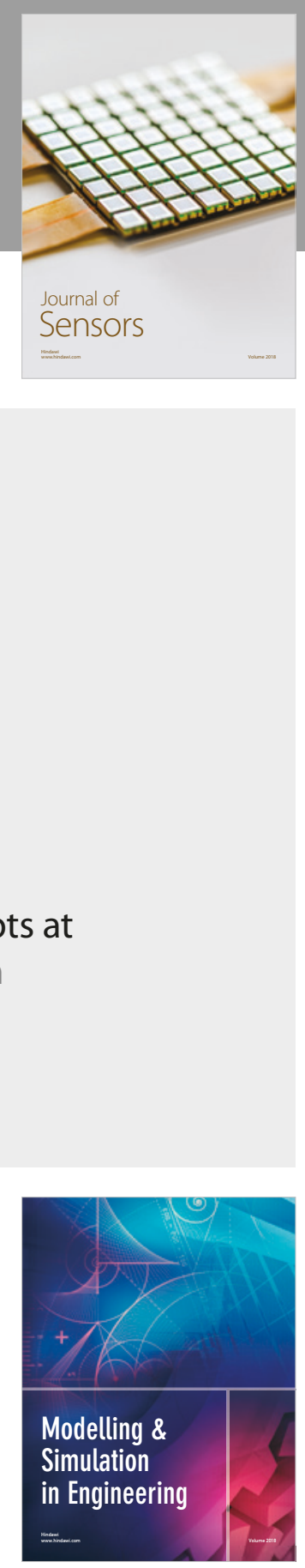

\section{Advances \\ Multimedia}
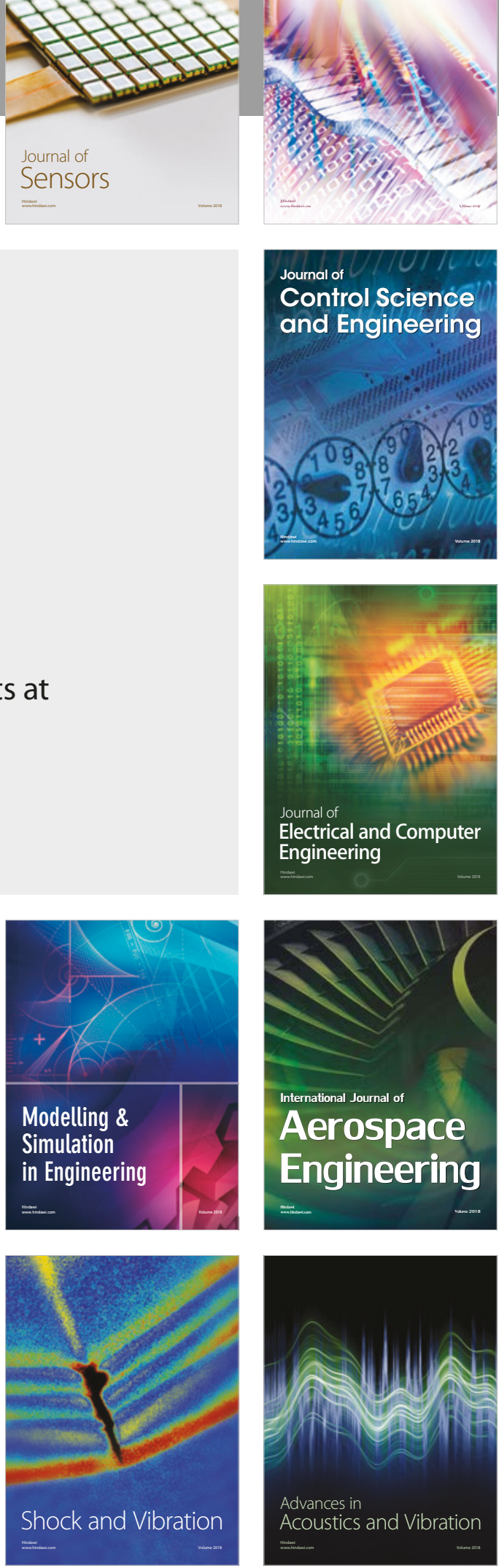